\title{
Sodium-Glucose Co-Transporter 2 Inhibitors (SGLT2i) Exposure and Outcomes in Type 2 Diabetes: A Systematic Review of Population-Based Observational Studies
}

\author{
Thomas M. Caparrotta (D) - Andrew M. Greenhalgh • Karen Osinski • \\ Robert M. Gifford · Svenja Moser · Sarah H. Wild • Rebecca M. Reynolds • \\ David J. Webb · Helen M. Colhoun
}

Received: November 13, 2020 / Accepted: January 13, 2021 / Published online: March 4, 2021

(C) The Author(s) 2021

\section{ABSTRACT}

Introduction: Sodium-glucose co-transporter 2 inhibitors (SGLT2is) are licensed for the treatment of type 2 diabetes (T2D) and more recently for heart failure with or without diabetes. They have been shown to be safe (from the cardiovascular (CV) perspective) and

Supplementary Information The online version contains supplementary material available at https:// doi.org/10.1007/s13300-021-01004-2.

T. M. Caparrotta $(\varangle) \cdot$ S. Moser $\cdot$ H. M. Colhoun Institute of Genetics and Molecular Medicine, University of Edinburgh, Edinburgh, UK

e-mail: tom.caparrotta@igmm.ed.ac.uk

T. M. Caparrotta - A. M. Greenhalgh · K. Osinski R. M. Gifford

NHS Lothian, Edinburgh Royal Infirmary, Edinburgh, UK

A. M. Greenhalgh · R. M. Gifford

Defence Medical Services, Whittington Barracks, Whittington, Lichfield, UK

R. M. Gifford · R. M. Reynolds · D. J. Webb University of Edinburgh/British Heart Foundation Centre for Cardiovascular Science, Queen's Medical Research Institute, Edinburgh, UK

S. H. Wild

Usher Institute, University of Edinburgh, Old

Medical School, Teviot Place, Edinburgh, UK

H. M. Colhoun

Department of Public Health, NHS Fife, Hayfield

House, Hayfield Rd, Kirkcaldy, UK effective (in terms of glycaemia, and in some cases, in reducing CV events) in extensive randomised controlled trials (RCTs). However, there remain concerns regarding the generalisability of these findings (to those ineligible for RCT participation) and about non-CV safety. For effectiveness, population-based pharmacoepidemiology studies can confirm and extend the findings of RCTs to broader populations and explore safety, for which RCTs are not usually powered, in more detail.

Methods: A pre-planned and registered ((International PROSPEctive Register Of Systematic Reviews) PROSPERO registration CRD42019160792) systematic review of population-based studies investigating SGLT2i effectiveness and safety, following Meta-analyses Of Observational Studies in Epidemiology (MOOSE) guidelines was conducted.

Results: A total of 37 studies were identified (total $n=1,300,184$ adults; total follow-up 910,577 person-years; exposures: SGLT2i class, canagliflozin, dapagliflozin and empagliflozin) exploring CV disease (CVD) outcomes, acute kidney injury (AKI), lower limb amputation (LLA), diabetic ketoacidosis (DKA), bone fracture, urinary tract infection (UTI), genital mycotic infection (GMI), hypoglycaemia, pancreatitis and venous thromboembolism. For CV and mortality outcomes, studies confirmed the associated safety of these drugs and correlated closely with the findings from RCTs, which may extend to primary CVD prevention (major 
adverse cardiovascular events point estimate range (PER) hazard ratio (HR) 0.78-0.94; hospitalised heart failure PER HR 0.48-0.79). For safety outcomes, SGLT2i exposure was not associated with an increased risk of AKI (PER HR 0.40-0.96), fractures (PER HR 0.87-1.11), hypoglycaemia (PER HR 0.76-2.49) or UTI (PER HR 0.72-0.98). There was a signal for increased association for GMIs (PER HR 2.08-3.15), and possibly for LLA (PER HR 0.74-2.79) and DKA (PER HR 0.96-2.14), but with considerable uncertainty.

Conclusion: In T2D, SGLT2is appear safe from the $\mathrm{CV}$ perspective and may have associated benefit in primary as well as secondary CVD prevention. For safety, they may be associated with an increased risk of GMI, LLA and DKA, although longer follow-up studies are needed.

Keywords: Comparative effectiveness; Observational studies; SGLT2 inhibitors; Systematic review; Type 2 diabetes

\section{Key Summary Points}

\section{Background}

Sodium-glucose co-transporter 2 inhibitors (SGLT2is) are licensed for the treatment of type 2 diabetes (T2D) and more recently for heart failure in those with and without diabetes.

In T2D, both canagliflozin and empagliflozin reduce major adverse cardiovascular events (MACE) but dapagliflozin does not. All three agents reduce heart failure in established diabetes and dapagliflozin reduces heart failure in those without diabetes. Furthermore, safety concerns have emerged, either during trials or through post-marketing surveillance, such as SGLT2i exposure possibly being associated with LLA, DKA, bone fracture and GMI.
For effectiveness, population-based pharmacoepidemiology studies can confirm and extend the findings of randomised controlled trials (RCTs) to broader populations not eligible for trial participation and explore safety, for which RCTs are not usually powered, in more detail.

\section{Why carry out this study?}

We did a pre-planned and registered, impartial systematic review asking: do the benefits of SGLT2is in T2D extend to those ineligible for RCT participation, and are safety concerns which arose during the trials (or in post-marketing) detected, in population-based observational pharmacoepidemiology studies? We considered and reported all clinical eventbased outcomes for effectiveness and safety in studies which met our inclusion/ exclusion criteria.

\section{What was learned from the study?}

A total of 37 population-based studies including adults $(n=1,300,184)$ with T2D were identified. These appear to confirm that SGLT2is in T2D appear safe from the CVD perspective, and may have associated benefit in primary as well as secondary CVD prevention, particularly in HFassociated events. However, SGLT2i exposure may be associated with an increased risk of GMI, LLA and DKA, although longer follow-up studies are needed.

\section{DIGITAL FEATURES}

This article is published with digital features, including a summary slide, to facilitate understanding of the article. To view digital features for this article go to https://doi.org/10.6084/ m9.figshare.13567889. 


\section{INTRODUCTION}

Sodium-glucose co-transporter 2 inhibitors (SGLT2is) are licensed for the treatment of type 2 diabetes mellitus (T2D) when diet and lifestyle have not improved glycaemic control. They have also recently been licensed by both European Medicines Agency (EMA) and US Food and Drug Administration (FDA) for the treatment of heart failure (HF) with reduced ejection fraction in those with or without diabetes. In diabetes, they improve glycaemic control by causing glycosuria, natriuresis and osmotic diuresis. Their effect is independent of insulin and they are thought to exert beneficial effects through pleiotropic mechanisms beyond improved glycaemia, including favourable haemodynamic changes [1].

The clinical development programmes for these agents assessed the efficacy, cardiovascular (CV) safety and preliminary non-CV safety of the SGLT2is in T2D. Many have subsequently been assessed for cardiovascular disease (CVD) safety in large trials and have been shown to be non-inferior, and sometimes superior, to usual care in this respect [2-6]. Both canagliflozin [5] and empagliflozin [2] reduce major adverse cardiovascular events (MACE) but dapagliflozin [3] does not. All three agents reduce HF in those with T2D [2, 3, 5] and dapagliflozin reduces HF in those without diabetes [4]. Almost all outcome trial participants were on background metformin.

It remains unclear, however, whether the $\mathrm{CV}$ benefits of these medicines extend to people who were ineligible for the trials. Furthermore, safety concerns have emerged, either during trials or through post-marketing surveillance $[2,3,5-26]$. It is also unclear whether the beneficial effects extend to the SGLT2i class as a whole or whether this is limited to individual agents. For non-CV safety, population-based observational pharmacoepidemiology allows for rare, but severe, adverse events to be detected.

Population-based studies present more generalisable data which can expand upon the findings from randomised controlled trials (RCTs) but the lack of randomisation and blinding increases the risk of bias and confounding. However, population-based database studies, when properly designed and analysed, are associated with fewer systematic distortions, such as selection bias, compared to other forms of observational pharmacoepidemiology [27].

This study describes a pre-planned and prospectively registered impartial systematic review of population-based, observational studies examining effectiveness and safety of SGLT2is in T2D ((International PROSPEctive Register of Systematic Reviews) (PROSPERO) registration CRD42019160792, 03 December 2019). It must be noted that there is a broad diversity in outcomes, particularly for CVD, as well as the definitions of pre-existing CVD, which makes interpretation challenging.

The hypothesis was that the studies included in this review would show a treatment effectiveness or safety estimate associated with SGLT2i exposure of a similar direction and order of magnitude to that reported in RCTs (or, for safety, pharmacovigilance studies).

\section{METHODS}

We followed Meta-analyses Of Observational Studies in Epidemiology (MOOSE) guidelines for reporting. In brief, two search methodologies were employed (maximal and targeted search) in the following citation databases: Web of Science, OVID, Excerpta Medica dataBASE (EMBASE) and PUBMED using the search criteria listed in the supplementary material, which were agnostic to specific outcomes (Supplementary Table S1). Searches were conducted for studies published between November 2012 (date of licensing dapagliflozin) and December 2020. Duplicates were eliminated using each study's unique identifier. Two (medically qualified) reviewers (AG and TC) independently applied the inclusion and exclusion criteria (Table S2) first to the title and subsequently to the abstracts. We searched European Network of Centres for Pharmacoepidemiology and Pharmacovigilance (ENCePP), ClinicalTrials.gov and the European Union (EU) Post-Authorisation Studies (PAS) registers to identify further studies. 
Studies were excluded that only reported continuous clinical outcomes (e.g. blood pressure) or studies that only reported adherence or tolerability. We only included English-language publications because of resource limitations.

Each reviewer checked 10\% of the other's title and abstract screening with a pre-specified decision that less than 95\% agreement would mean that the entire list would be re-screened. Third-party arbitration of disputes was planned in the event of less than 95\% agreement. There was $100 \%$ agreement of included/excluded studies and arbitration was not necessary.

A standardised data extraction template was used to collect study data (Table S3).

The studies eligible for inclusion were scored for quality using the Downs and Black Checklist for Non-Randomised Studies [28], which has been shown to have good inter-rater reliability, but no score cut-off for inclusion/exclusion was employed (Table S4) [29]. Score discrepancies were handled in conference with the reviewers, with third-party arbitration planned if agreement was not achieved but this latter step was not necessary.

The references of included studies were searched manually to identify studies which may not have been included in original searches. All reported outcomes were included.

Studies were subsequently tabulated by outcome to compare effect estimates and relevant subgroup analyses (so studies may appear in multiple tables). Effect estimates and relevant subgroup analyses were compared between studies in separate tables for each outcome. Forest plots were used to compare safety outcomes.

We chose to describe studies with fewer than 5000 participants as small, those with 5000-20,000 participants as medium-sized and those with more than 20,000 as large. Confidence interval (CI) widths are described qualitatively. The associated effect estimate is defined as neutral when no effect can be discerned, and as an associated increase/decrease in risk when the bound of the CI does not cross unity.

Meta-analysis was not performed as a result of breaching meta-analytic standards (Berlin et al. [30]). Study design heterogeneity, low study numbers for individual outcomes and multiple representation of data sources influenced this decision. A funnel plot was considered to assess the risk of publication bias but there were too few studies per outcome to undertake this (Cochrane Library recommends more than 10 studies per outcome [31]) Thus, we present the findings of a narrative systematic review.

This article is based on previously conducted studies and does not contain any new studies with human participants or animals performed by any of the authors.

\section{RESULTS}

The search and application of inclusion/exclusion criteria identified 37 individual studies within the dates specified (Fig. 1, Preferred Reporting Items for Systematic Reviews and Meta-Analyses (PRISMA) diagram) including $n=1,300,184$ persons; total follow-up 910,577 person-years. Of these, 21 were funded by academic institutions, public bodies or charity/non-profit organisations [32-52] and 16 were funded by pharmaceutical companies [53-68]. We found six studies that had been prospectively registered with either https:// clinicaltrials.gov/ (five studies $[56,58,63$, $65,67]$, with two sharing a registration number $[56,58]$ ) or EU PAS Register (one study [52]).

Table S4 reports the quality score agreed by two independent reviewers (TC and AG). The scores ranged from 10 to 22 (the maximum achievable score would have been $29 / 31$ as none of the studies were randomised). None of the study designs as described appeared to have been affected by immortal time bias arising from an inappropriate start date to follow-up.

There was a preponderance of studies of the earliest SGLT2is to achieve market authorisation (dapagliflozin, empagliflozin, canagliflozin), with SGLT2is licensed later (ipragliflozin (licensed only in Japan) and ertugliflozin) only included in SGLT2i class studies.

Table S5 describes the Comparative-Effectiveness of Cardiovascular Outcomes in New Users of Sodium-Glucose Cotransporter-2 Inhibitors (CVD-REAL) programme. 


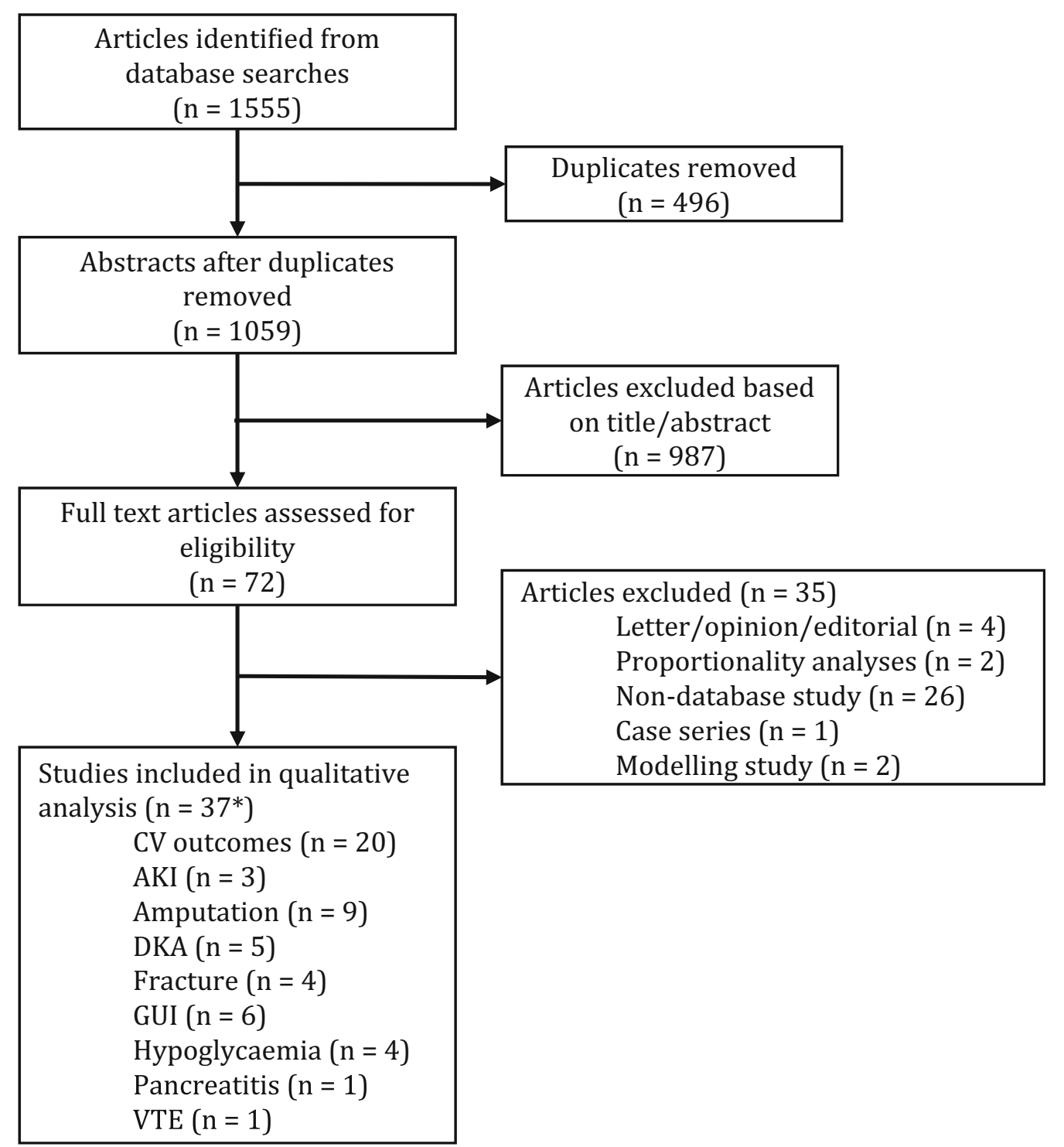

Fig. 1 PRISMA diagram

Tables 1, 2, 3, 4, 5, 6, 7, 8, 9 and 10 describe the studies in more detail. Tables S6-S10 and S14-S22 give the study characteristics and effect estimates.

Here we compare (and tabulate) the composites/outcomes together, grouped by exposure and comparator, to allow head-to-head contrasts to be drawn.

\section{Cardiovascular Disease}

\section{Non 3-Point MACE Cardiovascular Composites}

All-Cause Mortality (ACM) and Hospitalised Heart Failure (HHF) Composite Three studies explored the association of SGLT2i class exposure on the composite of ACM and HHF versus (vs.) oral anti-hyperglycaemic drugs (OADs) as a primary $[56,66]$ and secondary outcome [58]. All three studies were associated with a reduction in the composite and each component 
Table 1 CVD study summary

\section{Exposure/study Study summary \\ number}

SGLT2i class

1.1

1.2

1.3

1.4

1.5

1.6
The large 2017 main CVD-REAL cohort study explored the association of SGLT2i class exposure vs. OADs on HHF (primary outcome and composite component) [58]. SGLT2i exposure was associated with a reduction in the CVD composite of ACM and HHF (Table S6.3) and with ACM (Table S14.3) and HHF (Table S10.7) composite components alone with narrow CIs and authors suggest benefit may extend to primary prevention of HHF (although subgroup analysis on the basis of pre-existing CVD was not presented)

A large 2018 CVD-REAL sub-analysis explored the association of SGLT2i class vs. OAD on stroke and MI events [57]. SGLT2i exposure was associated with a reduction in the risk of stroke in all analyses (Table S9.6) and a neutral association with the risk of MI (reduced in the on-treatment cohort but not in the ITT cohort, Table S8.6) and suggests that the benefit may extend to primary prevention of these outcomes (although this subgroup analysis is not presented). The CIs were narrow

A further large 2017 CVD-REAL sub-analysis investigated the effect of SGLT2i class vs. OADs on CVD [54]. SGLT2i exposure was associated with a reduction in MACE (primary prevention-only, Table S7.2) and HF (primary and secondary prevention, Table S10.10), and a neutral association in non-fatal stroke and non-fatal MI (Tables S9.5 and S8.5) with no effect on the negative control, atrial fibrillation. The MACE benefit appeared greatest in those aged $\geq 65$ years (DNS) and CIs were narrow for all outcomes

CVD-REAL 2 was a large 2018 cohort study exploring the association of SGLT2i class exposure vs. OADs on HHF, MI and stroke [56]. SGLT2i exposure was associated with a reduction in HHF (Table S10.9), MI (Table S8.4), stroke (Table S9.4) and the composite of HHF and death

(Table S6.4) in all analyses. The CIs were narrow. The effect remained consistent in pre-planned subgroup analysis of those without pre-existing CVD (DNS)

A small 2017 American cohort study explored the association of SGLT2i class vs. DPP4i exposure on HHF [55]. SGLT2i exposure was associated with a reduction in HHF in the PS-matched, IPTW and MSM methods with narrow CIs (Table S10.4). This association was driven mainly by findings among people aged $\geq 65$ years and/or with pre-existing diabetes complications (DNS)

A large 2018 American cohort study explored the association between SGLT2i class vs. non-SGLT2i AHDs on MACE, HHF, non-fatal stroke and non-fatal MI [66]. SGLT2i exposure in the ITT analysis was associated with a reduction the primary composite outcome of ACM and HHF (Table S6.5), the HHF component alone (Table S10.6) and the secondary outcome of MACE overall (Table S7.3) but a neutral association with the non-fatal MI (Table S8.7) and non-fatal stroke (Table S9.7) components when analysed individually. The associated effects reported in the ITT analysis were amplified in the on-treatment analysis (DNS). All CIs were narrow. The results remained consistent in all the pre-planned subgroup analyses, including with and without preexisting CVD, suggesting an associated benefit in both primary and secondary prevention (DNS) 
Table 1 continued

Exposure/study Study summary
number

A large 2018 South Korean cohort study investigated the association of SGLT2i class exposure vs. DPP4i on HHF [40]. SGLT2i exposure was associated with a reduction in HHF in all time periods analysed up to 3 years post-initiation in the whole population and in the secondary prevention population with varying $\mathrm{CI}$ width (a duration-response effect) but not in the primary prevention population with the associated reduction in HHF only apparent in time periods $>1$ year post-exposure (lagged duration-response effect) (Table S10.5)

A medium-sized 2018 American cohort study explored the association of SGLT2i class vs. DPP4i on HHF, stroke, IHD and hospitalised PVD (negative control, schizophrenia) [62]. SGLT2i exposure was associated with a neutral effect for all outcomes including negative control (Tables S6.2, S10.3, S9.2, S8.1). The CIs were wide. These findings remained stable irrespective of baseline $\mathrm{HbAlc}$ and prior metformin use and also in the maximally adjusted and time-varying model (DNS)

A large 2019 American cohort study explored the association of SGLT2i class vs. SUs and DPP4is [37]. Versus SUs, SGLT2i exposure was associated with a lower risk of MI, HHF and stroke with a reduction also observed in the composite of MI and stroke. Versus DPP4is, SGLT2i exposure was associated with a reduction in HHF, MI, stroke with a reduction also observed in the composite of MI and stroke, all CIs were narrow (all comparisons in Tables S6.1, S10.1, S9.1, S8.3). The associated effect persisted in sensitivity analyses in those aged $<65$ years but not those aged $\geq 65$ years, but the associated reduction in CVD and HHF remained when the primary/ secondary CVD prevention groups and individual agents were analysed separately (DNS)

A medium-sized 2019 Scandinavian cohort study explored the association of SGLT2i class vs. DPP4i on primary/secondary prevention of MACE, HHF, incident MI and stroke [44]. SGLT2i use in the ITT analysis was associated with a reduction in the risk of HHF (in those with and without a history of HF but only in those with pre-existing CVD, when analysed separately) and also an associated neutral effect on MACE and its components (in both primary/secondary CVD prevention) (Tables S10.2, S9.3, S8.2, S7.1). CIs were narrow. The on-treatment analysis was associated with a reduction in MACE, but not in AMI and stroke (driven by a reduction in CVD mortality) while the reduction in HF was amplified (DNS). Individuals aged $\geq 65$ years saw an associated reduction in both MACE and HHF versus those aged $<65$ years (DNS), who did not with a diagnosis of HF, using loop-diuretic prescriptions as a proxy for HF symptomatic severity [51]. SGLT2i exposure was associated with a reduction in new loop-diuretic use but no change in diuretic use in those already exposed (Table S10.8) 
Table 1 continued

Exposure/study Study summary
number

Empagliflozin

A medium-sized 2019 American cohort study explored the association of empagliflozin vs. sitagliptin on HHF [63]. In the population overall there was a reduction in HHF associated with empagliflozin exposure, greater in the broad definition of HHF (HHF mentioned anywhere on discharge, DNS) compared to the specific one (HHF in primary position of discharge, Table S10.15). In subgroup analyses (pre-existing CVD, HF, sex and empagliflozin dose), the associated reduction for the broad HHF definition remained stable and demonstrated a dose-response effect but not for the specific definition (CVD subgroups in Table S10.15, other DNS). The CIs were wide. There was no association with negative control outcome, seasonal flu vaccination

Canagliflozin

A large 2018 American study investigated the association of canagliflozin exposure vs. all nonSGLT2i users and also vs. select non-SGLT2i (DPP4i, GLP-1RA, TZD, SU and insulin) and head-to-head with other SGLT2is (followed by other all SGLT2is vs. non-SGLT2i) on the association with HHF [65]. See Table S13 for baseline characteristics in each of the datasets. In the on-treatment analysis, a reduced association with HHF was observed in canagliflozin vs. all non-SGLT2i comparison (primary outcome) with narrow CIs, canagliflozin vs. select nonSGLT2is comparison and SGLT2is vs. non-SGLT2is but not for canagliflozin head-to-head with other SGLT2is (Table S10.16). This attenuated in the ITT analysis (DNS). In those with established CVD (75\% of the post-index events), there was also an associated reduction with canagliflozin vs. AHDs but not head-to-head with other SGLT2is, suggesting a class effect (DNS)

A medium-sized 2018 American cohort study explored the association of canagliflozin vs. DPP4i, GLP-1RA and SUs (analysed separately) on HHF (primary event-based outcome and diuretic use as a proxy) and a CVD composite of MI and stroke (secondary outcome) (Tables S11 and S12) [45]. Canagliflozin was associated with a risk reduction for HHF (Table S10.12) and diuretic use in all comparisons and a neutral effect for the composite CVD endpoint (Table S6.6) and the individual components of the composite (MI Table S8.10; stroke Table S9.10, other DNS) in both primary and secondary CVD prevention. The CIs were narrow. The on-treatment and baseline HbAlc-adjusted analyses yielded the same results (DNS)

Dapagliflozin

An unmatched medium-sized 2019 Taiwanese cohort study explored the association of dapagliflozin head-to-head with empagliflozin on the composite of CV mortality, MI, stroke and HHF (negative control, incident AF) [47]. Dapagliflozin exposure was associated with a neutral effect on the composite (Table S6.10), MI and stroke, but was associated with a reduction in HHF compared with empagliflozin (MI Table S8.11; stroke Table S9.11; HHF Table S10.14). This associated reduction remained stable in standardised mortality analysis, the low dose group and also when adjusting for CVD risk, regardless other CVD drug exposure (DNS). Both drugs showed a neutral effect on the negative control. The composite CI was narrow, but the other outcomes CIs were of varying width 
Table 1 continued

\begin{tabular}{ll}
\hline $\begin{array}{l}\text { Exposure/study } \\
\text { number }\end{array}$ & Study summary \\
\hline 1.16 & A medium-sized 2018 CVD-REAL sub-analysis investigated the association of dapagliflozin vs. \\
& DPP4i on MACE [64]. The on-treatment analysis showed dapagliflozin was associated with a \\
& reduced risk of MACE (Table S7.4), MACE, unstable angina and HHF expanded composite \\
& (DNS) and HHF alone (Table S10.11). For the MACE components there was a neutral effect on \\
& non-fatal MI (Table S8.9) and non-fatal stroke (Table S9.9). The CIs were narrow throughout. \\
& There was also a reduced association with incident AF. The results remained stable in the ITT \\
& analysis but primary/secondary CVD prevention groups were not analysed separately (DNS) \\
A & medium-sized 2017 Swedish cohort study explored the effect of dapagliflozin vs. insulin's \\
& association with non-fatal CVD [61]. Dapagliflozin exposure was associated with a reduction of \\
& non-fatal CVD in the PS-matched and the adjusted model (DNS), for both the on-treatment \\
(PSM Table S6.7) and ITT analyses (DNS). The CIs were narrow. Primary/secondary CVD \\
prevention was not analysed \\
A small 2017 UK cohort study explored the association between dapagliflozin and unexposed \\
controls on incident CVD (secondary outcome) [49]. A neutral association (Table S6.8) on \\
incident CVD in the low-risk (of CVD) population was observed (incident CVD in the overall \\
population not assessed). The CIs were narrow \\
A medium-sized 2019 Swedish cohort study examined the association of dapagliflozin vs. OADs on \\
MACE, HHF, MI, stroke and AF [60]. Dapagliflozin was associated, in the ITT analysis, with a \\
lower risk of HHF (Table S10.13) but a neutral association on MACE (Table S7.5), MI \\
(Table S8.8), stroke (Table S9.8) and AF. The CIs were narrow. The associated on-treatment \\
effect was amplified such that MACE reduction became significant (DNS) \\
A medium-sized 2019 Scottish cohort study explored the association of dapagliflozin exposure vs. \\
non-exposure on CVD (coronary/cerebrovascular disease) [59]. This was a secondary analysis. \\
Dapagliflozin was associated with a neutral effect with narrow CIs on incident CVD in the \\
dose-response analysis (Table S6.9)
\end{tabular}

$A C M$ all-cause mortality, $A H D s$ all hyperglycaemic drugs, $A M I$ acute myocardial infarction, $C I$ confidence interval, $C V D$ cardiovascular disease, CVD-REAL Comparative-Effectiveness of Cardiovascular Outcomes in New Users of Sodium-Glucose Cotransporter-2 Inhibitors, DNS data not shown, DPP4i dipeptidyl peptidase 4 inhibitor, GLP-1RA glucagon-like peptide 1 receptor agonist, HbAIc glycated haemoglobin, $H H F$ hospitalised heart failure, $I H D$ ischaemic heart disease, IPTW inverse probability of treatment weighting, ITT intention to treat, MACE major adverse cardiovascular event, $M I$ myocardial infarction, $M S M$ marginal structural model, $O A D$ oral anti-hyperglycaemic drug, $P V D$ peripheral vascular disease, $S G L T 2 i$ sodium-glucose co-transporter 2 inhibitor, $S U$ sulfonylurea, $T Z D$ thiazolidinedione

alone (Tables 1.1, 1.4, 1.6, S6.3, S6.4 and S6.5). There was less prevalent ischaemic heart disease (IHD) compared to the cardiovascular outcome trials (CVOTs) (ca. 13.0\% [58] and ca. 26.7\% [56] established IHD (not reported in [66]) compared to $45-75 \%[2,3,5])$ and in two studies the association remained consistent when secondary CVD prevention was examined alone in subgroup analysis $[56,66]$. 
Table 2 Mortality study summary

\section{Exposure/study Study summary \\ number}

SGLT2i class

2.1

Canagliflozin

Dapagliflozin

2.8
The main large 2017 CVD-REAL cohort study explored the association of SGLT2i class exposure vs. OADs on ACM [58]. SGLT2i exposure was associated with a reduction in ACM alone with narrow CIs (Table S14.3) and also in the composite with HHF (Table S6.3), stable geographically

A large 2017 Scandinavian study (CVD-REAL sub-analysis) explored the association of CVD mortality and ACM in SGLT2i class-exposed vs. OADs [54]. Authors report an association of lower CVD mortality in those exposed to SGLT2i (Table S14.4) in those with and without established CVD, with a greater reduction in those aged $>65$ years (DNS). There was also a reduced association with ACM (Table S14.4). The CIs were narrow

A large 2018 American cohort study explored the association between SGLT2i class vs. non-SGLT2i AHAs on ACM [66]. SGLT2i exposure was associated with a reduction in ACM (and also in the composite of ACM and HHF, Table S6.5) in the ITT cohort (Table S14.5), stable in the preplanned subgroup analysis (DNS). The CIs were narrow

The large 2018 CVD-REAL 2 cohort study exploring the association of SGLT2i class exposure vs. OADs on ACM [56]. SGLT2is were associated with a reduction in the ITT (Table S14.6) and on-treatment analyses, in those with and without established CVD groups (DNS) for ACM alone (Table S6.4). The CIs were narrow

A medium-sized 2019 Scandinavian cohort study explored the association of SGLT2i class vs. DPP4i on CVD mortality [44]. SGLT2is showed a neutral association with CVD mortality in the population overall in the ITT analysis (Table S14.1) but a reduced association in the on-treatment analysis (DNS). For ACM, SGLT2i use was associated with a reduction in the risk of ACM in the ITT (Table S14.1) and on-treatment groups (DNS). The CIs were narrow

A medium-sized 2019 Israeli case-control explored the association of SGLT2i class exposure vs. DPP4i on ACM [34]. This study showed an associated reduction in the odds of ACM in both the crude (DNS) and the adjusted model for SGLT2is (secondary analysis, Table S14.2). The CIs were wide

A medium-sized 2018 American cohort study comparing canagliflozin exposure pairwise with DPP4i, GLP-1RAs and SUs showed a neutral effect of CVD mortality in all comparisons (Table S14.9). These associations remained stable when adjusted for baseline HbAlc level (DNS) [45]. For ACM, there was a neutral association with canagliflozin (part of CVD composite) overall (Table S14.9) and in primary/secondary CVD prevention. These associations remained stable when adjusted for baseline HbAlc level (DNS). The CIs were wide for all comparisons

A medium-sized 2017 Swedish study explored the effect of dapagliflozin vs. insulin's association with ACM [61]. Dapagliflozin exposure was associated with a reduced risk of ACM in both those with, with narrow CIs, and without pre-existing CVD, with wider CIs than those with CVD (Table S14.10) 
Table 2 continued

\begin{tabular}{ll}
\hline $\begin{array}{l}\text { Exposure/study } \\
\text { number }\end{array}$ & Study summary \\
\hline 2.9 & A small 2017 UK cohort study explored the association between dapagliflozin and unexposed \\
controls on ACM [49]. There was an associated reduction in ACM in the both the low-risk (of \\
CVD) population and the population overall for dapagliflozin with narrow CIs (including those \\
with prevalent CVD) (Table S14.11) \\
A medium-sized 2019 Swedish cohort study examined the association between dapagliflozin vs. non- \\
SGLT2i OADs on CVD mortality and ACM [60]. Dapagliflozin exposure was associated with a \\
lower risk of CVD mortality (MACE component) in both the ITT (Table S14.7) and on- \\
treatment groups (DNS). For ACM, dapagliflozin was associated with a lower risk of ACM in \\
both analyses (ITT, Table S14.7). The CIs were narrow \\
A medium-sized 2018 CVD-REAL sub-analysis investigated the effect of dapagliflozin vs. DPP4i on \\
CVD mortality (MACE component) and ACM (single outcome) [64]. This study showed a \\
neutral association on CVD mortality in the on-treatment analysis (Table S14.8). For ACM there \\
was a reduced association (Table S14.8). The results for both outcomes remained stable in \\
subgroup/sensitivity analysis (DNS). The CIs were narrow \\
A \\
A medium-sized 2019 Taiwanese cohort study explored the association of dapagliflozin head-to-head \\
with empagliflozin for CVD mortality [47]. Dapagliflozin showed a neutral association with CVD \\
mortality ((Table S14.12) as part of a composite outcome), with wide confidence intervals, \\
(stable in sensitivity analyses, DNS). The CIs were wide
\end{tabular}

$A C M$ all-cause mortality, $A H A$ antihyperglycemic agent, $C I$ confidence interval, $C V D$ cardiovascular disease, $C V D-R E A L$ Comparative-Effectiveness of Cardiovascular Outcomes in New Users of Sodium-Glucose Cotransporter-2 Inhibitors, $D N S$ data not shown, DPP4i dipeptidyl peptidase 4 inhibitor, GLP-1RA glucagon-like peptide 1 receptor agonist, HbAIc glycated haemoglobin, $H H F$ hospitalised heart failure, ITT intention to treat, $M A C E$ major adverse cardiovascular event, $O A D$ oral anti-hyperglycaemic drug, $S G L T 2 i$ sodium-glucose co-transporter 2 inhibitor, $S U$ sulfonylurea

Myocardial Infarction (MI) and Stroke Composite Two studies investigated the composite of MI and stroke. For the SGLT2i class pairwise (head-to-head) vs. sulfonylureas (SUs) and DPP4is as a primary outcome there was an associated reduction in the composite for both comparisons (established CVD ca. 12\% compared to $45-75 \%[2,3,5]$ in the CVOTs, Tables 1.9, S6.1) [37]. For canagliflozin pairwise vs. DPP4is, glucagon-like peptide 1 receptor agonists (GLP-1Ras) and SUs there was a neutral effect on a CVD composite and for the individual components in all three comparisons (established CVD ca. $11 \%$ vs. $73 \%$ [5] in the CVOT, Tables 1.14, S6.6, S11, S12) [45]. There was also a neutral effect in the expanded composite and in secondary CVD prevention subgroup.

Other Composites For SGLT2i class vs. DPP4is, a study explored the composite of HHF, IHD, stroke and peripheral arterial disease (PAD) as a primary outcome (baseline CVD 4.3-5.5\% compared to $45-75 \%[2,3,5]$ in the CVOTs) and reported a neutral association in the population overall, and in primary/secondary CVD prevention (Tables 1.8 and S6.2) [62].

Dapagliflozin exposure was compared in four studies to OAD [49], non-users [59], insulin [61] and head-to-head with empagliflozin [47]. Versus $\mathrm{OAD}$, exposure was associated with a neutral effect on an incident CVD composite as a 
Table 3 Renal outcomes summary

\begin{tabular}{|c|c|}
\hline $\begin{array}{l}\text { Exposure/study } \\
\text { number }\end{array}$ & Study summary \\
\hline \multicolumn{2}{|l|}{ SGLT2i class } \\
\hline 3.1 & $\begin{array}{l}\text { A medium-sized } 2018 \text { Scandinavian study using Sweden and Denmark's population registers, } \\
\text { analysed separately, explored SGLT2i class vs. GLP-1RAs' (only Sweden for the maximally } \\
\text { adjusted model) association with AKI [50]. Both the unadjusted and maximally adjusted HR } \\
\text { showed a neutral association for AKI in those exposed to SGLT2i but both CIs were wide } \\
\text { (Table S15.1, Fig. 2). The CIs were moderately wide. In subgroups analysis, the effects remained } \\
\text { stable except in those with pre-existing CVD, which was associated with a reduction in AKI } \\
\text { (DNS) }\end{array}$ \\
\hline 3.2 & $\begin{array}{l}\text { A medium-sized } 2019 \text { Israeli study explored the deterioration of CKD status and hospitalisation for } \\
\text { AKI in those exposed to SGLT2i class vs. DPP4i [34]. SGLT2i exposure was associated with a } \\
\text { reduced odds of the composite of hospitalisation with AKI, initiation of dialysis and sustained } \\
\text { eGFR }<15 \text { but with no effect on deterioration of CKD category (Table S15.2, Fig. 2). The CIs } \\
\text { were narrow }\end{array}$ \\
\hline 3.3 & $\begin{array}{l}\text { A small } 2017 \text { American cohort study compared the use of SGLT2i class vs. OAD, in two separate } \\
\text { datasets, analysed separately, on the association with AKI [43]. Two definitions of AKI (KDIGO } \\
\text { and ICD) were used and SGLT2i exposure was associated a reduction AKI in the MS dataset } \\
\text { (adjusted and unadjusted) but not the smaller GHS dataset, where the associated effect attenuated } \\
\text { in the adjusted model (Table S15.3, Fig. 2). The CIs were narrow. These results remained } \\
\text { stable when SGLT2i drugs were analysed individually }\end{array}$ \\
\hline
\end{tabular}

$A K I$ acute kidney injury, $C K D$ chronic kidney disease, $C I$ confidence interval, $C V D$ cardiovascular disease, $D N S$ data not shown, DPP4i dipeptidyl peptidase 4 inhibitor, $e G F R$ estimated glomerular filtration rate, GHS Geisinger Health System, GLP-1RA glucagon-like peptide 1 receptor agonist, $H R$ hazard ratio, ICD International Classification of Diseases, $K D I G O$ Kidney Disease: Improving Global Outcomes, MS Mount Sinai chronic kidney disease registry, $S G L T 2 i$ sodium-glucose cotransporter 2 inhibitor

secondary outcome (MI, IHD, stroke/TIA, HHF and left ventricular dysfunction; baseline IHD $13.2-14.8 \%$ compared to $40.5 \%$ [3] in the CVOT; Tables 1.18 and S6.8.) in a low-risk of CVD population ( $\sim$ primary prevention) [49]. Versus non-users, exposure was associated with a neutral effect on a composite of IHD, cerebrovascular disease, HHF, cardiac arrhythmias or coronary revascularisation, as a secondary outcome (baseline IHD not reported, Tables 1.20 and S6.9) [59]. Versus insulin, exposure was associated with a reduction of the composite of fatal and non-fatal CVD as a primary outcome (MI, stroke, UA, HHF and CVD death; prior MI $9 \%$ vs. $40.5 \%$ any atherosclerotic history [3] from the CVOT, Tables 1.17 and
S6.7) [61]. Head-to-head with empagliflozin, exposure was associated with a neutral effect on the composite (as a primary outcome) of MI, stroke and HHF (baseline IHD $14.3 \%$ vs. $40.5 \%$ [3] in CVOT, Tables 1.15 and S6.10) [47].

\section{MACE}

MACE was defined as a composite of mortality (we allowed the mortality definition to be both CV mortality and ACM), non-fatal MI and nonfatal stroke ( \pm other outcomes, reported separately).

Three studies explored the association with MACE for the SGLT2i class in patients with lower baseline CVD $(6-25 \%[44,54,66])$ than 
Table 4 Amputation study summary

\section{Exposure/study Study summary \\ number}

SGLT2i class

4.1

4.2

4.3

4.4

4.5

4.6
A large 2019 American cohort study compared SGLT2i class exposure vs. DPP4is and SUs on the risk of LLA [37]. This study reported that compared to SUs, SGLT2i use was associated with a lower risk of LLA but neutral compared to DPP4is (Table S16.1, Fig. 3). The CIs were narrow

A medium-sized 2018 American cohort study compared the hazard of LLA associated with SGLT2i class exposure vs. DPP4is and SUs [52]. The study reports an elevated association of LLA compared to DPP4i and a neutral association compared to SUs in all analyses with wide CIs for both comparisons. Overall, $83 \%$ of the amputations were of the toe and metatarsal (Table S16.2, Fig. 3). In subgroup analysis, characteristics associated with an increased LLA risk were history of amputation, baseline insulin use and history of CKD in both analyses (but metformin use and history of CVD-only in the SU comparison, DNS). Results remained stable in sensitivity analysis, expect for canagliflozin alone, which had a higher HR (DNS)

A medium-sized 2018 American cohort study investigated the association of SGLT2i class vs. DPP4i with LLA [33]. The maximally adjusted HR suggests SGLT2i exposure was associated a neutral effect on risk of LLA (Table S16.3, Fig. 3). The CIs were wide. Three-quarters of detected amputations occurred at the level of partial foot. In subgroup analysis, there was a neutral association for increased risk in those aged $\geq 65$ years, with pre-existing PVD or with at $\geq 1$ vascular complication of diabetes ('high-risk group', DNS). The risk of amputation was higher in the dapagliflozin or empagliflozin group than the canagliflozin group and the results remained stable in sensitivity analysis (DNS)

A medium-sized 2019 Scandinavian study compared association of LLA (as a supplementary analysis) in SGLT2i class vs. DPP4i [44]. The study reports a neutral association (Table S16.4, Fig. 3). The CIs were wide

A medium-sized 2018 American study investigated the association of SGLT2i class vs. GLP-1RAs and DPP4i with LLA with a neutral association in both comparisons (Table S16.5, Fig. 3) [35]. The CIs were wide. Compared to users of older T2D drugs (metformin, TZDs and SUs), SGLT2i exposure was associated with an increased risk of vascular ulcers, osteomyelitis and PVD but not compared to DPP4is/GLP-1RA (DNS). Results remained stable in sensitivity analysis, except that when those with prior amputation were included SGLT2i exposure was associated with increased risk (DNS)

A medium-sized 2018 Scandinavian study explored the association of SGLT2i class vs. GLP-1RA for LLA [50]. There was an increased associated risk of amputation overall (but not toe and metatarsal amputation) in both the whole population (Sweden and Denmark) and the maximally adjusted model (Sweden only) (Table S16.6, Fig. 3). The CIs were wide. In subgroup analysis, the associations remained stable geographically and for sex, although there was an associated increased risk in those ages $<65$ years and in those with pre-existing CVD (but not previous amputation, DNS) 
Table 4 continued

\begin{tabular}{|c|c|}
\hline $\begin{array}{l}\text { Exposure/study } \\
\text { number }\end{array}$ & Study summary \\
\hline 4.7 & $\begin{array}{l}\text { A large } 2018 \text { American cohort study investigated the association of SGLT2i class vs. non-SGLT2i } \\
\text { with incident BKA [66]. There was an associated increased risk of BKA in the ITT group (DNS) } \\
\text { and a neutral association risk of BKA in the on-treatment group, although the CIs were wide } \\
\text { (Table S16.7, Fig. 3). Subgroup analyses suggest an associated elevated risk of BKA in men, those } \\
\text { on insulin, those not exposed to GLP-1RA, those with } \geq 2 \mathrm{CV} \text { risk factors and in those with pre- } \\
\text { existing renal disease (CIs wider still, DNS) }\end{array}$ \\
\hline \multicolumn{2}{|l|}{ Canagliflozin } \\
\hline 4.8 & $\begin{array}{l}\text { A large } 2018 \text { American cohort study investigated the association between canagliflozin vs. OAD } \\
\text { (excluding metformin) on BKA [68]. Authors report a neutral association for risk of amputation } \\
\text { (Table S16.8, Fig. 3). The CIs were wide. The results remained stable in sensitivity analysis (DNS) }\end{array}$ \\
\hline \multicolumn{2}{|l|}{ Dapagliflozin } \\
\hline 4.9 & $\begin{array}{l}\text { A medium-sized } 2019 \text { Scottish study cohort study sought to determine a dose-response effect of } \\
\text { dapagliflozin exposure (vs. non-exposure) on LLA [59]. Dapagliflozin was associated with a neutral } \\
\text { risk of amputation but with wide CIs (Table S16.9, Fig. 3) }\end{array}$ \\
\hline
\end{tabular}

$B K A$ below-knee amputation, $C I$ confidence interval, $C K D$ chronic kidney disease, $C V D$ cardiovascular disease, $D N S$ data not shown, DPP4i dipeptidyl peptidase 4 inhibitor, GLP-1RA glucagon-like peptide 1 receptor agonist, $H R$ hazard ratio, $I T T$ intention to treat, $L L A$ lower limb amputation, $O A D$ oral anti-hyperglycaemic drug, $P V D$ peripheral vascular disease, $S G L T 2 i$ sodium-glucose co-transporter 2 inhibitor, $S U$ sulfonylurea, $T 2 D$ type 2 diabetes, $T Z D$ thiazolidinedione

the CVOTs $(45-75 \%[2,3,5])$ but with inconsistent findings. One study (vs. OAD) showed a surprising associated reduction in the primary CVD prevention subgroup but not in the secondary prevention subgroup or the population overall, where the largest expected benefit might be in those with pre-existing CVD (Tables 1.3, S7.2) [54]. Versus OAD (SGLT2i non-users) exposure was associated with a reduction in MACE (secondary outcome) in both the intention-to-treat (ITT) and on-treatment analysis and also in primary/secondary CVD prevention subgroups (Tables 1.6, S7.3) [66]. Versus DPP4is, there was an associated reduction in MACE in the on-treatment but not in the ITT analysis (consistent in primary/secondary CVD prevention) (Tables 1.10, S7.1) [44].

For dapagliflozin versus dipeptidyl peptidase 4 inhibitor (DPP4i) (CVD-REAL sub- analysis, 23\% pre-existing CVD), a reduced association for MACE was demonstrated (and for the expanded composite) in both the ITT and on-treatment analyses (Tables 1.16, S7.4) [64]. Versus OAD (a CVOT inclusion/exclusion emulation for dapagliflozin, but with 10\%-less established CVD), exposure was associated with a reduction in MACE in the on-treatment, but not the ITT, analysis, driven by a reduction in CV mortality (Tables 1.19, S7.5) [60].

\section{Myocardial Infarction}

MI was always a composite component.

For the SGLT2i class vs. OAD (CVD-REAL sub-analysis, 13\% established CVD), exposure was associated with a decrease in MI events (ontreatment and ITT analyses) with benefit in primary/secondary CVD prevention (Tables 1.2, 
Table 5 DKA study summary

\begin{tabular}{|c|c|}
\hline $\begin{array}{l}\text { Exposure/study } \\
\text { number }\end{array}$ & Study summary \\
\hline \multicolumn{2}{|l|}{ SGLT2i class } \\
\hline 5.1 & $\begin{array}{l}\text { A medium-sized } 2018 \text { Scandinavian study explored the association of SGLT2i class vs. GLP-1RA on } \\
\text { DKA [50]. SGLT2i exposure was associated with a neutral risk of DKA in both unadjusted } \\
\text { (Sweden and Denmark) and the maximally adjusted population (Sweden only) but with very wide } \\
\text { CIs (Table 11.1, Fig. 4). In subgroup analysis, those aged }<65 \text { years appeared to be at increased } \\
\text { risk of DKA vs. older users (DNS) }\end{array}$ \\
\hline 5.2 & $\begin{array}{l}\text { A large } 2019 \text { South Korean study comparing SGLT2i class exposure vs. DPP4i on DKA risk [ } 41] \text {. } \\
\text { Authors report neutral association for DKA (with no evidence of a duration-response effect, } \\
\text { Table S17.2, Fig. 4) and narrow CIs. Subgroup analysis in those with microvascular disease and } \\
\text { those exposed to diuretics had a higher associated HR but remained non-significant (DNS) }\end{array}$ \\
\hline 5.3 & $\begin{array}{l}\text { A medium-sized } 2019 \text { Scandinavian study also explored the association of SGLT2i class vs. DPP4i } \\
\text { on DKA (supplementary analysis) [44]. There was a positive association with DKA (Table S17.3, } \\
\text { Fig. 4). The CIs were wide }\end{array}$ \\
\hline 5.4 & $\begin{array}{l}\text { A medium-sized } 2017 \text { US study compared SGLT2i class vs. OADs (excluding metformin) on DKA } \\
\text { [67]. There was a neutral association for DKA but with extremely wide CIs (broad definition, } \\
\text { DNS), which attenuated with a more specific definition of T2D (Table S17.4, Fig. 4). The specific } \\
\text { definition attempted to exclude possible T1D; this reduced the analysis population by } 10 \% \text { but } \\
\text { DKA events by }>50 \% \text {. In the per-protocol sensitivity analysis, SGLT2i exposure was associated } \\
\text { with a significant risk of DKA under the broad definition but not in the specific definition (DNS) }\end{array}$ \\
\hline \multicolumn{2}{|l|}{ Dapagliflozin } \\
\hline 5.5 & $\begin{array}{l}\text { A medium-sized } 2019 \text { Scottish study explored the association of dapagliflozin vs. non-exposure on } \\
\text { the rates of DKA, seeking a dose-response effect [59]. There was an associated neutral DKA risk } \\
\text { but the CIs were wide (Table S17.5, Fig. 4) }\end{array}$ \\
\hline
\end{tabular}

$C I$ confidence interval, DKA diabetic ketoacidosis, DNS data not shown, DPP4i dipeptidyl peptidase 4 inhibitor, GLP$I R A$ glucagon-like peptide 1 receptor agonist, $H R$ hazard ratio, $O A D$ oral anti-hyperglycaemic drug, SGLT2i sodium-glucose co-transporter 2 inhibitor, $T 2 D$ type 2 diabetes

S8.6) [57]. This contrasts with another CVDREAL sub-analysis (SGLT2i vs. OAD) with a neutral association on MI (Tables 1.3, S8.5) [54]. There was a reduced association in CVD-REAL 2 (geographically distinct to CVD-REAL, vs. OAD, $27 \%$ established CVD at baseline) for MI in the ITT, on-treatment (OT) and adjusted analyses (overall and in primary/secondary CVD prevention) (Tables 1.4, S8.4) [56] A further study revealed a neutral association, vs. OAD, for MI (secondary outcome) in ITT (but reduced in OT analysis), and stable in primary/secondary CVD prevention subgroup (Tables 1.6, S8.7) [66].
Versus DPP4i there was a neutral association in two studies [44, 62] for MI/IHD; for one study both in ITT (Tables 1.10, S8.2) and OT analyses [44]; and for the other study the association remained stable regardless of baseline glycated haemoglobin (HbA1c) and metformin use (Tables 1.8, S8.1) [62]. Whereas versus DPP4i or SUs, there was a negative association for MI in both comparisons, persisting in primary/secondary CVD prevention subgroups but individuals aged less than 65 years benefitting more (Tables 1.9, S8.3) [37]. 
Table 6 Bone fracture study summary

\begin{tabular}{|c|c|}
\hline $\begin{array}{l}\text { Exposure/study } \\
\text { number }\end{array}$ & Study summary \\
\hline \multicolumn{2}{|l|}{ SGLT2i class } \\
\hline 6.1 & $\begin{array}{l}\text { A medium-sized 2018, Scandinavian study investigated the association of SGLT2i class vs. GLP- } \\
\text { 1RAs on fracture risk [50]. Both the unadjusted (Sweden and Denmark) and the maximally } \\
\text { adjusted population (Sweden-only) data showed an associated increased fracture risk, with narrow } \\
\text { CIs (Table S18.1, Fig. 5). This association remained stable when hospitalised fractures and } \\
\text { osteoporotic fractures were analysed separately (DNS) }\end{array}$ \\
\hline 6.2 & $\begin{array}{l}\text { A medium-sized } 2019 \text { German nested, 1:40 matched, case-control study explored the association of } \\
\text { metformin + SGLT2i class vs. metformin }+ \text { any OAD on upper- and lower-limb fracture [46]. } \\
\text { There was a neutral association in both the crude (DNS) and adjusted analysis (Table S18.2, } \\
\text { Fig. 5), with narrow CIs. Subgroup analysis by fracture site, SGLT2i dose, falls and CVD remained } \\
\text { stable (DNS) }\end{array}$ \\
\hline \multicolumn{2}{|l|}{ Canagliflozin } \\
\hline 6.3 & $\begin{array}{l}\text { A large } 2019 \text { American cohort study assessed the association between canagliflozin vs. GLP-1RAs on } \\
\text { the risk of fractures in two data sources combined [38]. Canagliflozin was associated with a neutral } \\
\text { risk of fracture, both when the data sources were analysed separately (DNS) and combined } \\
\text { (Table S18.3, Fig. 5) with narrow CIs. There was a neutral association with an elevated risk of } \\
\text { fracture in subgroup/sensitivity analysis (DNS) }\end{array}$ \\
\hline \multicolumn{2}{|l|}{ Dapagliflozin } \\
\hline 6.4 & $\begin{array}{l}\text { A small 2018, UK study cohort study investigated the association of dapagliflozin exposure vs. } \\
\text { dapagliflozin-unexposed persons on any fracture risk [48]. The study reported a neutral association } \\
\text { of dapagliflozin on fragility fractures (defined by site: vertebral, humerus, proximal femoral, distal } \\
\text { radius; DNS) and all fractures (Table S18.4, Fig. 5). The CIs were narrow. The results remained } \\
\text { stable in sensitivity analysis in those at higher risk of fracture (DNS) }\end{array}$ \\
\hline
\end{tabular}

$C I$ confidence interval, $C V D$ cardiovascular disease, $D N S$ data not shown, GLP-1RA glucagon-like peptide 1 receptor agonist, $O A D$ oral anti-hyperglycaemic drug, $S G L T 2 i$ sodium-glucose co-transporter 2 inhibitor, T2D type 2 diabetes

For canagliflozin vs. DPP4i, GLP-1RAs and SUs there was a neutral association for MI in all pairwise comparisons (Tables 1.14, S8.10, S11, S12) [45].

For dapagliflozin (vs. DPP4i, CVD-REAL subanalysis) there was a neutral association with MI (Tables 1.16, S8.9) [64], and similar in another study, vs. OADs, where there was neutral association in all analyses (Tables 1.19, S8.18) [60]. A head-to-head comparison vs. empagliflozin showed a neutral association with MI (Tables 1.15, S8.11) [47].

\section{Stroke}

Non-fatal stroke was always a composite component.

For SGLT2i class vs. OADs (CVD-REAL subanalysis) there was an associated reduction in stroke (ITT and OT analysis, Tables 1.2, S9.6) [57], although another CVD-REAL sub-analysis suggests a neutral association (Tables 1.3, S9.5) [54]. For CVD-REAL 2 study, vs. OADs, there was a reduced association for stroke (all analyses) (Tables 1.4, S9.4) [56]. Versus OADs, there 
Table 7 Genitourinary infections (UTI/GMI)

\section{Outcome/exposure/study Study summary \\ number}

Urinary tract infection

7.1

7.2

7.3

7.4

Canagliflozin

7.5

Genital mycotic infection

SGLT2i class

7.6

7.7
A medium-sized 2018 Scandinavian study explored the association of SGLT2i class vs. GLP1RA on UTI [50]. There was a neutral association for UTI in whole population and also in the maximally adjusted (Sweden only) population with narrow CIs (Table S19.1, Fig. 6), stable in all subgroup analyses (DNS)

A large 2019 American cohort study explored the association of SGLT2i class vs. GLP-1RA and DPP4i on UTI [36]. SGLT2is were associated with lower risk of UTI vs. GLP-1RA but neutral vs. DPP4is with narrow CIs (Table S19.2, Fig. 6), consistent in subgroup and sensitivity analysis (DNS)

A small 2017 Australian cohort study explored the association of SGLT2i class vs. DPP4i exposure on UTI [39]. There was a neutral association for UTI (Table S19.3, Fig. 6) with moderately wide CIs

A medium-sized 2019 Canadian cohort study explored the association for UTI in older people comparing SGLT2i class exposure vs. DPP4i, seeking a duration-response effect [42]. There was a reduced association with UTI at 30, 90 and 120 days post-initiation overall, with narrow CIs (Table S19.4, Fig. 6; 120 days, other DNS) and in both men and women when analysed separately (DNS)

A medium-sized 2017 American cohort study explored the association of canagliflozin exposure vs. non-canagliflozin AHAs on the risk of UTI [53]. There was a neutral association of canagliflozin on UTI with narrow CIs (Table S19.6, Fig. 6), stable in sensitivity analysis (DNS)

A small 2017 Australian study investigated the association of SGLT2i class vs. DPP4i exposure on GMI [39]. There was an increased association for GMI (Table S19.3, Fig. 7) but with wide CIs

A medium-sized 2019 Canadian study explored the association of SGLT2i class vs. DPP4i on GMI, seeking a duration-response effect [42]. There was an elevated associated risk of GMI, sustained over time, in the overall population with narrow CIs (Table S19.4, Fig. 7; 120 days, other DNS) and in both men and women when analysed separately, stable in sensitivity analysis (DNS) 
Table 7 continued

\begin{tabular}{|c|c|}
\hline $\begin{array}{l}\text { Outcome/exposure/study } \\
\text { number }\end{array}$ & Study summary \\
\hline 7.8 & $\begin{array}{l}\text { A medium-sized } 2018 \text { American study explored the (within-person) association of SGLT2i } \\
\text { class with GMI using a prescription symmetry analysis (antifungal prescription rates as } \\
\text { proxy for GMI) before and after SGLT2i initiation [32]. There was an associated increased } \\
\text { risk of antifungal prescription at } 30,60,90,180 \text { and } 365 \text { days post-initiation, suggesting } \\
\text { sustained risk (Table S19.5 for } 365 \text { days, other DNS). The association remained in } \\
\text { subgroup analysis for both men and women (but women at higher risk, DNS). } \\
\text { Canagliflozin was associated with a higher risk of an antifungal prescription compared to } \\
\text { dapagliflozin and empagliflozin exposure (individual drug analysis, DNS) }\end{array}$ \\
\hline \multicolumn{2}{|l|}{ Canagliflozin } \\
\hline 7.9 & $\begin{array}{l}\text { A medium-sized } 2017 \text { American cohort study explored the association of canagliflozin vs. } \\
\text { non-canagliflozin controls with GMI [53]. There was an increased association overall for } \\
\text { GMI (Table S19.6, Fig. 7); but when analysed separately, the association remained for } \\
\text { women but not for men (DNS). The CIs were narrow }\end{array}$ \\
\hline
\end{tabular}

$A H A$ antihyperglycemic agent, $C I$ confidence interval, $D N S$ data not shown, DPP $4 i$ dipeptidyl peptidase 4 inhibitor, GLPIRA glucagon-like peptide 1 receptor agonist, GMI genital mycotic infection, $S G L T 2 i$ sodium-glucose co-transporter 2 inhibitor, UTI urinary tract infection

was a neutral association (ITT analysis, marginally beneficial in the OT) (Tables 1.6, S9.7) [66].

Versus DPP4i and SUs, for class exposure, there was a reduced association for stroke for both comparisons, persisting in subgroup analysis (Tables 1.9, S9.1) [37]. Versus DPP4i, exposure was associated with a neutral effect for primary/secondary CVD prevention (Tables 1.8, S9.2) [62], a finding replicated in another study with the same comparison (both ITT and OT analysis) (Tables 1.10, S9.3) [44].

For canagliflozin (versus DPP4i, GLP-1RA and SUs, pairwise) a neutral association with stroke was observed (Tables 1.14, S9.10, S11, S12) [45].

For dapagliflozin head-to-head with empagliflozin there was a neutral association with stroke (Tables 1.15, S9.11) [47]. Versus DPP4is, there was a neutral association with stroke risk, stable in subgroup/sensitivity analysis (Tables 1.16, S9.9) [64]. Versus DPP4i (CVD-
REAL sub-analysis) a neutral association for stroke was observed [64], which was similar in another study (vs. OADs, ITT and OT) (Tables 1.19, S9.8) [60].

\section{Heart Failure}

HF was explored either alone, as part of a composite or with proxies.

For SGLT2i class, vs. OAD, both CVD-REAL and CVD-REAL 2 main analyses showed an associated reduction in HHF, holding in sensitivity/subgroup analysis (Tables 1.3, S10.10; Tables 1.4, S10.9; Tables 1.1, S10.7) [54, 56, 58]. Versus OADs there was an associated reduction in HHF (in both those with and without baseline HF, Tables 1.6, 4.7) [66]. As a proxy for HF, versus $\mathrm{OAD}$, there was an associated reduction in incident loop-diuretic prescriptions following exposure but not for dose change/prescription cessation (Tables 1.11, S10.8) [51]. 
Table 8 Hypoglycaemia study summary

\section{Exposure/study Study summary \\ number}

SGLT2i class

8.1

A large 2017 Scandinavian CVD-REAL sub-analysis explored the association of SGLT2i class vs. other OAD [54]. This study showed that compared to OADs, SGLT2i exposure use was associated with a lower risk of hospitalised severe hypoglycaemia (Table S20.1, Fig. 8). The CIs were narrow

Dapagliflozin

A medium-sized 2018 Scandinavian CVD-REAL sub-analysis explored the association of dapagliflozin vs. DPP4i exposure for hospitalised severe hypoglycaemia (as sub-analysis) [64]. There was a neutral association with hypoglycaemia (Table S20.2, Fig. 8). The CIs were narrow

A medium-sized 2019 Scandinavian study examined the association between dapagliflozin vs. OADs for hospitalised severe hypoglycaemia (as sub-analysis) [60]. There was a neutral association for hypoglycaemia (Table S20.3, Fig. 8), with narrow CIs

A medium-sized 2017 Swedish study explored the association of dapagliflozin vs. insulin on hospitalised severe hypoglycaemia (sub-analysis) [61]. There was a neutral association in both the PSM (Table S20.4, Fig. 8) and the multivariate-adjusted model (DNS). The CIs were wide

$A C M$ all-cause mortality, $C I$ confidence interval, CVD-REAL Comparative-Effectiveness of Cardiovascular Outcomes in New Users of Sodium-Glucose Cotransporter-2 Inhibitors, DNS data not shown, DPP4i dipeptidyl peptidase 4 inhibitor, $O A D$ oral anti-hyperglycaemic drug, $P S M$ propensity score-matched, $S G L T 2 i$ sodium-glucose co-transporter 2 inhibitor

Table 9 Pancreatitis study summary study

\section{Outcome/exposure/study Study summary number}

Pancreatitis

9.1

One medium-sized 2018 Scandinavian study explored the association of SGLT2i class vs.

GLP-1RA exposure on the pancreatitis [50]. There was a neutral association with pancreatitis in the whole population (Sweden and Denmark, DNS), the maximally adjusted population (Sweden only) with wide CIs (Table S21) and in subgroup analysis (DNS)

$C I$ confidence interval, $D N S$ data not shown, GLP-1RA glucagon-like peptide 1 receptor agonist, $S G L T 2 i$ sodium-glucose co-transporter 2 inhibitor

Versus DPP4is, SGLT2i class exposure (13.7\% baseline atherosclerosis) was associated with a reduction in HHF (population overall, individuals aged 65 years or more, and those with preexisting diabetes complications; Tables 1.5, S10.4) [55] and in the same comparison in another study (ca. 60\% baseline HF) for both primary/secondary HF prevention (after at least 3 years in the former, Tables 1.7, S10.5) [40]. This pattern repeated in another study (vs. DPP4i, all analyses) with the greatest associated reduction in individuals aged 65 years or more (Tables 1.10, S10.2) [44]. Compared to DPP4is (and SUs) there was a reduced association in 
Table 10 Venous thromboembolism study summary study

\begin{tabular}{|c|c|}
\hline $\begin{array}{l}\text { Outcome/exposure/study } \\
\text { number }\end{array}$ & Study summary \\
\hline \multicolumn{2}{|l|}{ Venous thromboembolism } \\
\hline 10.1 & $\begin{array}{l}\text { One medium-sized } 2018 \text { Scandinavian study explored the effect of SGLT2i class vs. GLP-1RA } \\
\text { for VTE [50]. This study showed a neutral association for VTE in the whole population } \\
\text { (Sweden and Denmark, DNS), the maximally adjusted population with narrow CIs } \\
\text { (Sweden-only, Table S22) and subgroup analysis (DNS) }\end{array}$ \\
\hline
\end{tabular}

$C I$ confidence interval, $D N S$ data not shown, GLP-1RA glucagon-like peptide 1 receptor agonist, $S G L T 2 i$ sodium-glucose co-transporter 2 inhibitor, VTE venous thromboembolism

both comparisons for HHF (12.6\% baseline IHD, and for primary/secondary CVD prevention, Tables 1.9, S10.1) [37]. Versus DPP4is (with lower baseline CVD, 5.5\%), a neutral association was observed in both primary/secondary HF prevention, static in sensitivity analysis (Tables 1.8, S10.3) [62].

For empagliflozin, vs. sitagliptin (25\% prevalent CVD), an associated reduction was demonstrated for HHF (primary outcome, Tables 1.12, S10.12) [63].

For canagliflozin (compared to all hyperglycaemic drugs (including injectable therapies) (AHDs), $0.3-1.4 \%$ prevalent HF), there was an associated reduction in HHF vs. non-SGLT2i drugs but not head-to-head with other SGLT2is (Tables 1.13, S10.16, S13) [65]. Versus DPP4is, GLP-1RAs and SUs, pairwise, canagliflozin was associated with reduced HHF (all analyses) (Tables 1.14, S10.12, S11, S12) [45].

Head-to-head, dapagliflozin vs. empagliflozin was associated with a reduced risk of HHF in all analyses and adjustments (Tables 1.15, S10.14) [47]. Versus DPP4is, exposure was associated with reduced HHF (CVD-REAL sub-analysis) (Tables 1.16, S10.11) [64] and also compared to OADs (Tables 1.19, S10.13) [60], in all analyses.

\section{Mortality}

CVD mortality was always a composite component. ACM was reported as part of a composite and also alone as an outcome.
SGLT2i class exposure, vs. OAD (CVD-REAL sub-analysis), was associated with a reduction in CVD mortality (main driver of MACE reduction; Tables 2.2, S14.4) [54]. Versus DPP4is, SGLT2i exposure was associated with a neutral effect on CVD mortality (Tables 2.5, S14.1) [44]. Compared to $\mathrm{OAD}$, there was an associated reduction in ACM in four studies, in two as a primary outcome (Tables 2.2, S14.4; Tables 2.1, S14.3) $[54,58]$, and for two as a component of a composite in all analyses (Tables 2.4, S14.6; Tables 2.3, S14.5) $[56,66]$. Compared to DPP4is, SGLT2i exposure was associated with a reduction in the risk of ACM in two studies (Tables 2.6, S14.2; Tables 2.5, S14.1) [34, 44].

For canagliflozin (vs. DPP4i, GLP-1RA and SUs, compared pairwise), exposure was associated with a neutral effect on CVD mortality across all comparisons (Tables 2.7, S14.9, S11, S12) [45]. Compared to DPP4i, GLP-1RAs and SUs there was a neutral association with ACM as a primary outcome in all pairwise comparisons [45].

Compared to empagliflozin, dapagliflozin exposure was associated with a neutral effect on CVD death (Tables 2.12, S14.12) [47] and also vs. DPP4is (Tables 2.11, S14.8) [64] and OADs (Tables 2.10, S14.7) [60].

Dapagliflozin was associated with a reduction in ACM compared to DPP4is in a CVDREAL sub-analysis [64] and compared to nondapagliflozin controls (in both the high- and low-risk of CVD populations; Tables 2.9, S14.11) [49], compared to insulin (in both OT and ITT analyses; Tables 2.8, S14.10) [61] and compared 
to OADs, as part of a composite, in both the ITT and OT analysis (Tables 2.10, S14.7) [60].

\section{Renal Outcomes (Acute Kidney Injury)}

For SGLT2i class vs. OADs exposure was associated with a reduction of acute kidney injury (AKI) in the unadjusted models but this effect attenuated in the adjusted models (stable when the analysis was repeated for individual agents and for both AKI definitions, Kidney Disease: Improving Global Outcomes (KDIGO) and International Classification of Diseases (ICD); Tables 3.3, S15.3) [43]. Versus GLP-1RAs, there was a neutral association of SGLT2i exposure (ICD definition, adjusted and unadjusted models) reduced in those with baseline CVD in subanalysis (Tables 3.1, S15.1) [50]. SGLT2i exposure vs. DPP4i was associated with a reduction in the composite renal outcome (hospitalisation for AKI, starting dialysis or sustained reduction in estimated glomerular filtration rate (eGFR) $<15 \mathrm{~mL} / \mathrm{min} / 1.73 \mathrm{~m}^{2}$ ) but with no association in deterioration of chronic kidney disease (CKD) category (Tables 3.2, S15.2) [34].

The renal benefit of SGLT2i exposure, demonstrated in RCTs, appears to be replicated in these studies, with no elevation in association with AKI [34, 43, 50], although the CIs were wide and all crossed unity and follow-up time was short, suggesting these studies may be underpowered.

Figure 2 shows the forest plot for these outcomes.

\section{Amputation}

For the SGLT2i class four studies have a point estimate suggesting an increased association with amputation but with considerable uncertainty. Compared to OADs subgroup analysis (ITT) suggests an elevated association in men, those on insulin, those not exposed to GLP1RAs, those with two or more CVD risk factors and those with pre-existing renal disease (Tables 4.7, S16.7) [66]. Versus GLP-1RAs, there was an associated elevated risk with subgroup, suggesting pre-existing CVD as a risk factor (but not in other subgroups; Tables 4.6, S16.6) [50].
Compared to DPP4is (but not SUs) exposure was associated with an increased risk of lower-limb amputation (LLA) (mainly toe and metatarsal loss), with baseline amputation, insulin use and CKD highlighted as particular risk factors (Tables 4.2, S16.2) [52]. Versus DPP4is and GLP1RAs there was a neutral association with LLA (Tables 4.5, S16.5). Exposure was associated with an elevated risk of vascular ulcers, osteomyelitis and peripheral vascular disease (PVD) (proxies for amputation) vs. metformin, thiazolidinediones (TZDs) and SUs, but not DPP4is/GLP-1RAs, data not shown (DNS)) [35]. The remaining three SGLT2i class studies suggest a neutral association with amputation compared to DPP4i (Tables 4.3, S16.3; Tables 4.1, S16.1; Tables 4.4, S16.4) [33, 37, 44] with previous amputation being a risk factor for future amputation in one study [33].

Canagliflozin was compared to non-SGLT2i agents, which showed a neutral association, although follow-up was short (Tables 4.8, S16.8) [68]. For dapagliflozin compared to non-dapagliflozin controls, exposure was associated with a neutral association for LLA (but the 95\% CIs were wide) (Tables 4.9, S16.9) [59].

For amputation, four comparisons had a neutral or reduced association [37, 52, 68], but only one study (vs. SU) did not cross unity [37]. Eight comparisons yielded an effect estimate suggesting an increased association of SGLT2i exposure with amputation [32, 35, 44, 50, 52, 59], with two comparisons not crossing unity (vs. DPP4i [52] and vs. GLP1RA [50]). Many of the CIs were wide, suggesting the studies may have been underpowered. On balance, there appears to be a signal for elevated LLA risk following SGLT2i exposure, but with significant uncertainty, which may be modulated by baseline characteristics and comparator drug chosen.

Figure 3 shows the forest plot for these outcomes.

\section{Diabetic Ketoacidosis}

Two studies found an increased association with SGLT2i exposure on diabetic ketoacidosis (DKA) (vs. GLP-1RAs Tables 5.1, S17.1 [50] and vs. 
DPP4i, as a supplemental analysis, Tables 5.3, S17.3 [44]). In the former study, no subgroup was at elevated risk except individuals aged less than 65 years [50].

Other studies found a neutral association with DKA exposure vs. DPP4i (with neutral but higher hazard ratios (HRs) in those with microvascular disease and on diuretics; Tables 5.2, S17.2) [41] and compared to OADs (Tables 5.4, S17.4) [67].

Finally, for dapagliflozin (vs. non-dapagliflozin controls) a neutral association was observed for DKA but with wide 95\% Cis (Tables 5.5, S17.5) [59].

Only one study (vs. GLP-1RA) showed an increased association for DKA but with very wide confidence intervals [50]. Of the other four studies, three had an effect estimate suggesting positive association $[44,59,67]$ and one suggesting negative association [41]; three $[44,59,67]$ of these had wide CIs suggesting the studies may be underpowered.

Figure 4 shows the forest plot for these outcomes.

\section{Fracture}

Four studies explored the association of SGLT2i exposure on fracture risk [38, 46, 48, 50].

For SGLT2i class, vs. OADs, a neutral association was observed on fractures overall and in subgroup analysis (Tables 6.2, S18.2) [46]. Versus GLP-1RAs, SGLT2i exposure was associated with a neutral effect on all fractures and also major osteoporotic fractures (Tables 6.1, S18.1) [50]. For canagliflozin (vs. GLP-1RA) there was a neutral association with fracture risk stable in all analyses, although follow-up was short (Tables 6.3, S18.3) [38]. For dapagliflozin (vs. unexposed controls) a neutral association was observed on all fractures and also fragility fractures (Tables 6.4, S18.4) [48].

All of the confidence intervals crossed unity.

Figure 5 shows the forest plot for these outcomes.

\section{Genitourinary Infections}

\section{Urinary Tract Infections}

For SGLT2i class (vs. GLP-1RAs), a neutral association was observed on urinary tract infection (UTI) risk across all subgroups (Tables 7.1, S19.1) [50].

A study comparing the association of UTI risk of SGLT2i exposure to GLP-1RAs and DPP4is found a neutral association compared to GLP-1RA but a reduced risk compared to DPP4is (Tables 7.2, S19.2) [36]. Compared to DPP4is alone, SGLT2i exposure was also associated with a neutral risk of UTI (Tables 7.3, S19.3) [39]. However, another analysis comparing SGLT2i exposure to DPP4i (in older people) found a reduced association for UTI in both men and women (Tables 7.4, S19.4) [42]. Finally, for canagliflozin (vs. non-canagliflozin controls), a neutral association was observed for UTI and also in sensitivity analysis (Tables 7.5, S19.6) [53].

Figure 6 shows the forest plot for these outcomes.

\section{Genital Mycotic Infections}

For the SGLT2i class, vs. DPP4i, exposure was associated with an increased risk of genital mycotic infection (GMI) (Tables 7.6, S19.3) [39]. In the same comparison in a different study, exposure was associated with an increased risk in both men and women, which persisted over time (Tables 7.7, S19.4) [42]. In a prescription symmetry analysis (using antifungal prescriptions as a proxy for GMI), SGLT2i class exposure was associated with an increased risk of receiving a prescription for treatment of GMI in both men and women over time but with women receiving more treatment prescriptions (with the strongest association for canagliflozin) (Tables 7.8, S19.5) [32].

For canagliflozin, vs. non-canagliflozin controls, there was an elevated association of exposure with GMI overall, but when men and women were analysed separately only women had an elevated association (Tables 7.9, S19.6) [53].

All three studies which explored GMI showed a significantly elevated association of 
Table 11 Current RCT and other evidence related to outcomes

\begin{tabular}{|c|c|}
\hline Outcome & RCT evidence summary \\
\hline \multirow[t]{5}{*}{$\begin{array}{l}\text { Major adverse } \\
\text { cardiovascular events }\end{array}$} & $\begin{array}{l}\text { Both empagliflozin and canagliflozin reduce cardiovascular morbidity and mortality in patients } \\
\text { with T2D at high CVD risk, on the basis of CVOT data }\end{array}$ \\
\hline & For MACE \\
\hline & Empagliflozin, HR 0.86 (95\% CI 0.74, 0.99) \\
\hline & Canagliflozin, HR 0.86 (96\% CI 0.75, 0.97) \\
\hline & Dapagliflozin, HR 0.93 (95\% CI 0.84, 1.03) does not improve these outcomes $[2,3,5]$ \\
\hline \multirow[t]{5}{*}{ Myocardial infarction } & $\begin{array}{l}\text { For non-fatal MI, none of the CVOTs showed a significant reduction in this component of } \\
\text { the MACE composite }\end{array}$ \\
\hline & Empagliflozin, HR 0.87 (95\% CI 0.70, 1.09) \\
\hline & Canagliflozin, HR 0.85 (95\% CI 0.69, 1.05) \\
\hline & Dapagliflozin, HR 0.77 (95\% CI 0.77, 1.01) \\
\hline & $\begin{array}{l}\text { This was not a primary outcome meaning the trials were not specifically powered to detect this } \\
{[2,3,5]}\end{array}$ \\
\hline \multirow[t]{5}{*}{ Stroke } & $\begin{array}{l}\text { For non-fatal stroke, all of the CVOTs showed a neutral effect on this component of the } \\
\text { MACE composite }\end{array}$ \\
\hline & Empagliflozin, HR 1.24 (95\% CI 0.92, 1.67) \\
\hline & Canagliflozin, HR 0.90 (95\% CI 0.71, 1.15) \\
\hline & Dapagliflozin, HR 1.01 (95\% CI 0.84, 1.21) \\
\hline & $\begin{array}{l}\text { There was a trend towards an increased risk in the case of empagliflozin; this was not a } \\
\text { primary outcome and the trials were not powered to detect this }[2,3,5]\end{array}$ \\
\hline \multirow[t]{5}{*}{ Heart failure } & $\begin{array}{l}\text { For hospitalised HF all three CVOTs suggest that SGLT2i exposure leads to a reduction in } \\
\text { this outcome }\end{array}$ \\
\hline & Empagliflozin, HR 0.65 (95\% CI 0.50, 0.85) \\
\hline & Dapagliflozin, HR 0.73 (95\% CI $0.61,0.88$ ) \\
\hline & Canagliflozin, HR 0.67 (95\% CI 0.52, 0.87) $[2,3,5]$ \\
\hline & $\begin{array}{l}\text { The DAPA-HF trial, in patients with and without T2D with baseline HF, showed a } \\
\text { reduction for first worsening of HF, CVD mortality and ACM [4] }\end{array}$ \\
\hline \multirow[t]{4}{*}{ Mortality } & The CVOT trials returned the following results for mortality outcomes \\
\hline & $\begin{array}{l}\text { Empagliflozin: CVD mortality, HR } 0.62 \text { (95\% CI 0.49, 0.77); ACM, HR } 0.68 \text { (95\% CI 0.57, } \\
0.82 \text { ) }\end{array}$ \\
\hline & $\begin{array}{l}\text { Canagliflozin: CVD mortality, HR } 0.87 \text { (95\% CI 0.72, 1.06); ACM, HR } 0.87 \text { (95\% CI 0.74, } \\
1.01 \text { ) }\end{array}$ \\
\hline & $\begin{array}{l}\text { Dapagliflozin: CVD mortality, HR } 0.98 \text { (95\% CI 0.82, 1.17); ACM, HR } 0.93 \text { (95\% CI 0.82, } \\
1.04)[2,3,5]\end{array}$ \\
\hline
\end{tabular}


Table 11 continued

Outcome

Renal outcomes

Amputation

Diabetic ketoacidosis

\section{RCT evidence summary}

Post-marketing reports to the FDA have suggested that both canagliflozin and dapagliflozin are associated with AKI, particularly after immediate treatment initiation. This is multifactorial because of volume depletion, hypotension or on concomitant nephrotoxic drugs [69]. However, both CVOTs and RCTs designed to test the effect on SGLT2is in diabetic nephropathy suggest that empagliflozin, canagliflozin and dapagliflozin are associated with a reduced incidence of worsening nephropathy (although the benefit may be reduced in those with more severe renal disease at baseline, possibly via a direct renovascular effect) [70]

Empagliflozin showed a reduction in the composite of progression of microalbuminuria, doubling of serum creatinine, initiation of RRT or death from renal causes, HR 0.61 (95\% CI $0.53,0.70)[71]$

For canagliflozin, there was a reduction of the composite of ESRD, doubling of serum creatinine or death from cardiovascular and renal causes, HR 0.70 (95\% CI 0.59, 0.82) [6]

In the case of dapagliflozin, exposure was associated with a reduction of the composite of $\geq 40 \%$ in eGFR, new ESRD or death from cardiovascular or renal causes, HR 0.76 (95\% CI $0.67,0.87)[3]$

SGLT2is, and particularly canagliflozin, have been associated with increased risk of amputation. Two RCTs investigating canagliflozin demonstrated a two-fold increase in the risk of (mainly toe and foot) amputation with those with PVD, neuropathy and prior amputation at the most elevated risk (IRR per 1000 patient-years, 5.9 vs. 2.8 (CANVAS) and 7.5 vs. 4.2 (CANVAS-R) [5]). Pharmacovigilance studies have also demonstrated an increased risk of amputation. Absolute numbers were low and the proportionality reporting ratio of adverse event descriptions may have been biased by knowledge of concern around SGLT2i exposure and amputation risk, making such events more likely to be reported in those taking SGLT2i [15]

DKA, often euglycaemic, has been reported in people taking SGLT2i agents, particularly canagliflozin. In all three CVOTs the numbers of DKA events were numerically higher in those exposed to SGLT2is but the absolute numbers were low

Empagliflozin: $n=4$ vs. 1

Canagliflozin: IRR 0.6 per 1000 patient-years vs. 0.3

Dapagliflozin: HR 2.18 (95\% CI 1.1, 4.3) [2, 3, 5]

Case series have also described associating SGLT2i exposure with an increased risk of DKA [23] 
Table 11 continued

\section{Outcome}

Bone fracture

\section{RCT evidence summary}

SGLT2i exposure, especially canagliflozin, has been associated with an increased fracture risk. Putative mechanisms include trauma following postural hypotension brought about by the volume-depleting effect of SGLT2is (increased risk soon after therapy initiation) or a reduction of BMD (which would lead to a slower increase in risk of fracture as bone mineral depletion necessarily takes time)

In RCTs involving canagliflozin some, but not all, demonstrated a higher risk of fracture in those exposed to canagliflozin $[6,25]$. For instance, a dose-response effect was demonstrated in patients taking $100 \mathrm{mg}$ and $300 \mathrm{mg}$ of canagliflozin (IRR 1.4 and 1.5 fracture per 100 patient-years) and was elevated compared to placebo (IRR 1.1 per 100 patient-years) [25]. In a BMD trial, canagliflozin, when compared to placebo, was associated with a progressive decline in BMD at the hip, with larger daily doses associated with a greater decline in BMD $[7,10]$

Trials of dapagliflozin on BMD did not show an effect [18] and neither did pooled trial analysis of fracture risk for empagliflozin [16]

Meta-analysis of safety outcomes did not find an increased fracture risk for empagliflozin and dapagliflozin, and the elevated OR for canagliflozin was not statistically significant, OR 1.15 (95\% CI 0.71, 1.88), although the source studies were not powered to detect fracture as an outcome [24]

Genitourinary infection

RCTs of SGLT2is show a two- to four-fold increase in the incidence of genitourinary candidiasis, with vulvovaginal candida infection occurring in 10-15\% of women exposed, compared to placebo $[8,22]$. Meta-analyses have also found that exposure to SGLT2is is associated with an increased risk of female genital candida infection [12, 19, 72]

Also, there have been descriptions of SGLT2is being associated with urological sepsis and pyelonephritis, as well as necrotising fasciitis of the perineal tissues $[9,14,17]$

Hypoglycaemia

SGLT2i are insulin-independent agents and are not associated with an elevated risk of hypoglycaemia when not co-prescribed with treatment that cause hypoglycaemia. They reduce the risk of hypoglycaemia compared to SUs and insulin, and hence may be indicated in people at high risk of hypoglycaemia (and weight gain); however, they may increase the risk of hypoglycaemia when co-prescribed with these other drugs [11, 20, 26]

Pancreatitis
SGLT2is have not been reported, in the literature, to cause an increased risk of pancreatitis. However T2D, in and of itself, is associated with an increased risk of pancreatitis compared to those without the condition $[13,21]$ 
Table 11 continued

\begin{tabular}{ll}
\hline Outcome & RCT evidence summary \\
\hline Venous thromboembolism & $\begin{array}{l}\text { SGLT2is have been putatively suggested to increase the risk of VTE, due to the } \\
\text { haemoconcentration occurring through the osmotic diuresis which they bring about. There } \\
\text { is no literature identified from RCTs to suggest an elevated risk of VTE }\end{array}$ \\
\hline
\end{tabular}

$A C M$ all-cause mortality, $A K I$ acute kidney injury, $B M D$ bone mineral density, $C I$ confidence interval, $C V D$ cardiovascular disease, CVOT cardiovascular outcomes trial, DKA diabetic ketoacidosis, eGFR estimated glomerular filtration rate, ESRD end-stage renal disease, $F D A$ US Food and Drug Administration, $H H F$ hospitalised heart failure, $H R$ hazard ratio, IRR incidence rate ratio, $M A C E$ major adverse cardiovascular event, $M I$ myocardial infarction, $O R$ odds ratio, $P V D$ peripheral vascular disease, $R C T$ randomised controlled trial, $R R T$ renal replacement therapy, $S G L T 2 i$ sodium-glucose co-transporter 2 inhibitor, $S U$ sulfonylurea, $T 2 D$ type 2 diabetes, $V T E$ venous thromboembolism

SGLT2i exposure with GMI [39, 42, 53]. There appears to be an increased association for both men and women, although women may be at greater risk.

Figure 7 shows the forest plot for these outcomes.

\section{Hypoglycaemia}

For the SGLT2i class, compared to OAD, exposure was associated with a reduced risk of hypoglycaemia (Tables 8.1, S20.1) [54]. For dapagliflozin, compared to insulin, the association was neutral, although the CIs were wide (Tables 8.4, S20.4) [61]. However, when compared to DPP4is (Table 8.2, S20.2) [64] and OAD (Tables 8.3, S20.3) [60] the associations observed were neutral.

Figure 8 shows the forest plot for these outcomes.

\section{Pancreatitis}

Although T2D itself raises the risk of pancreatitis, the one study we identified exploring the effect of SGLT2i exposure compared to GLP-1RA (which may be associated with increased risk) a neutral association was observed in all analyses (Tables 9.1, S21) [50].

\section{Venous Thromboembolism}

As a result of the diuretic effect of SGLT2i agents, it had been proposed that exposure may lead to haemoconcentration, and hence to elevated risk of venous thromboembolism (VTE). The one study we found exploring SGLT2i exposure (compared to GLP-1RAs) on VTE risk found a neutral association in the population overall and in all subgroups (Tables 10.1, S22) [50].

\section{DISCUSSION}

This study adds rich data on the CVD effectiveness of SGLT2i agents for T2D in broad populations, including people ineligible for CVOT participation, and examines a wide variety of safety outcomes. The CVOTs compared SGLT2i exposure to placebo (usual care). However, many of the studies we identified enable exploration of head-to-head comparative effectiveness. There was broad heterogeneity in outcomes, comparators and study design.

In order to contextualise the discussion Table 11 contains the evidence generated from RCTs (or other sources) related to the outcomes reported.

Quality scores ranged widely (10-22/31). No studies reported blinding their data analysts and few had power calculations. It was also often unclear whether subgroup analysis had been pre-planned or done post hoc. 


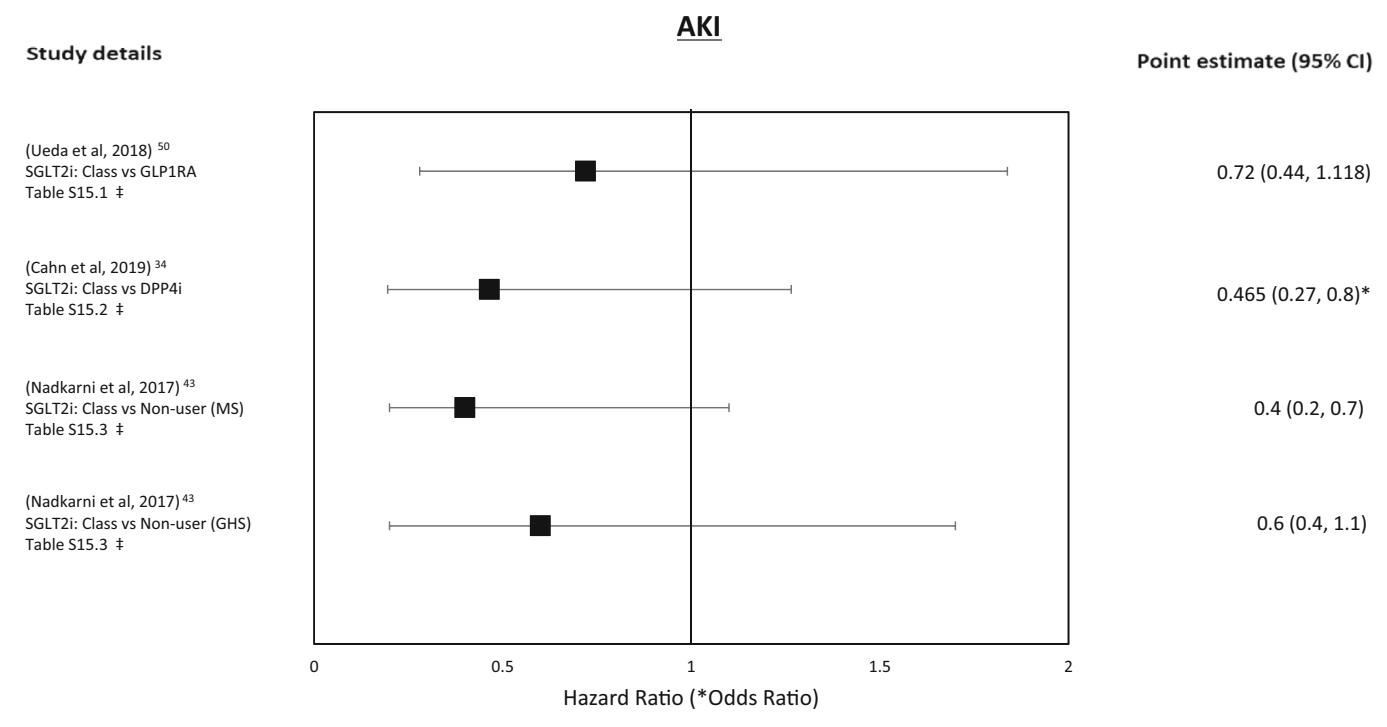

Fig. 2 Renal outcomes

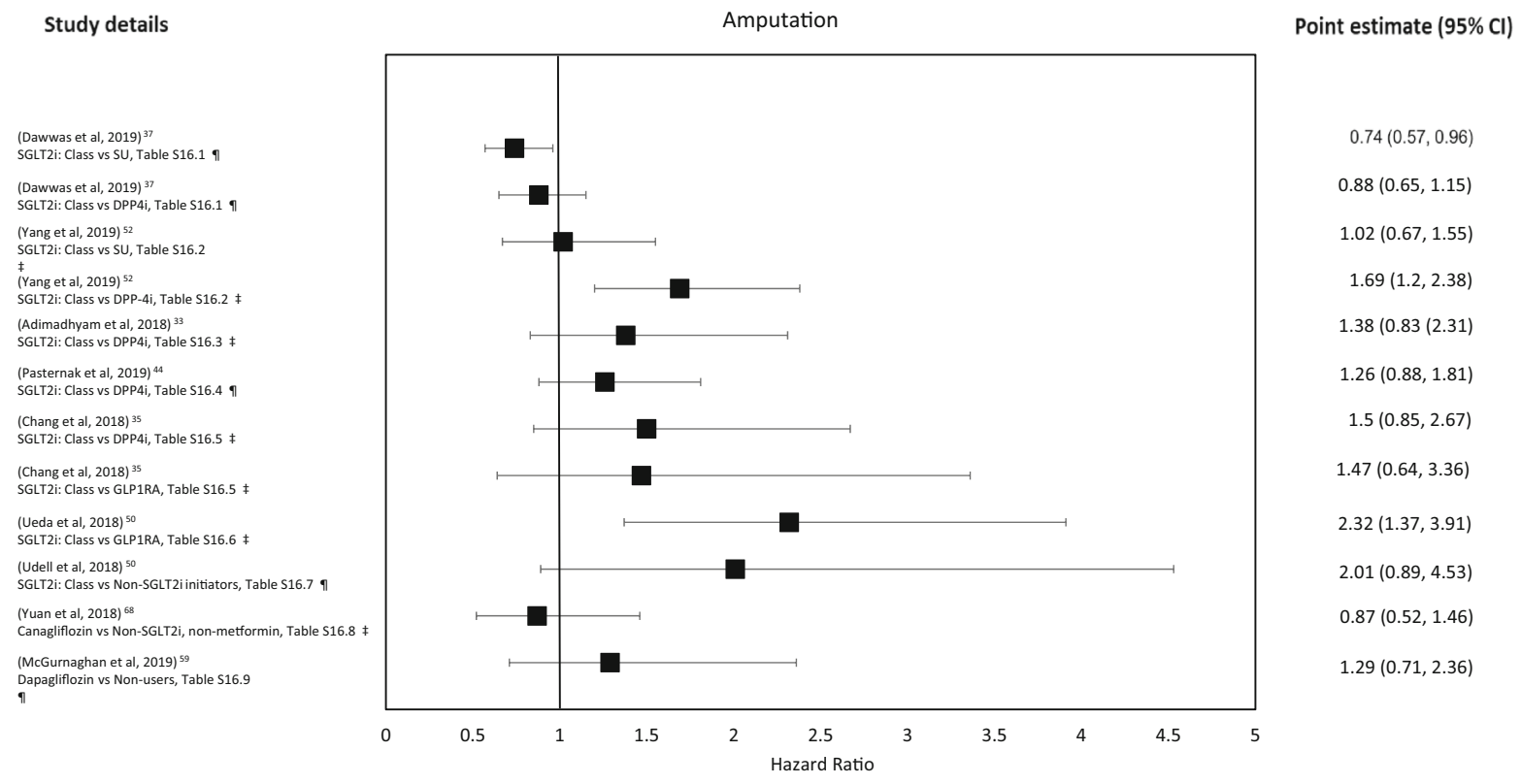

Fig. 3 Amputation

\section{Cardiovascular Disease and Mortality}

From RCT data, empagliflozin and canagliflozin reduce MACE, but dapagliflozin does not $[2,3,5]$. None of the agents reduce non-fatal MI or stroke [2, 3,5]. Only empagliflozin reduces $\mathrm{CV}$ mortality and ACM, the other agents reduce neither $[2,3,5]$. All three agents reduce HHF $[2,3,5]$.

No studies we found were associated with an increased risk of CVD, suggesting CV safety. The studies we found were either associated with a reduction in CVD or a neutral effect. Multiple (but not all) studies suggest that this benefit 
DKA

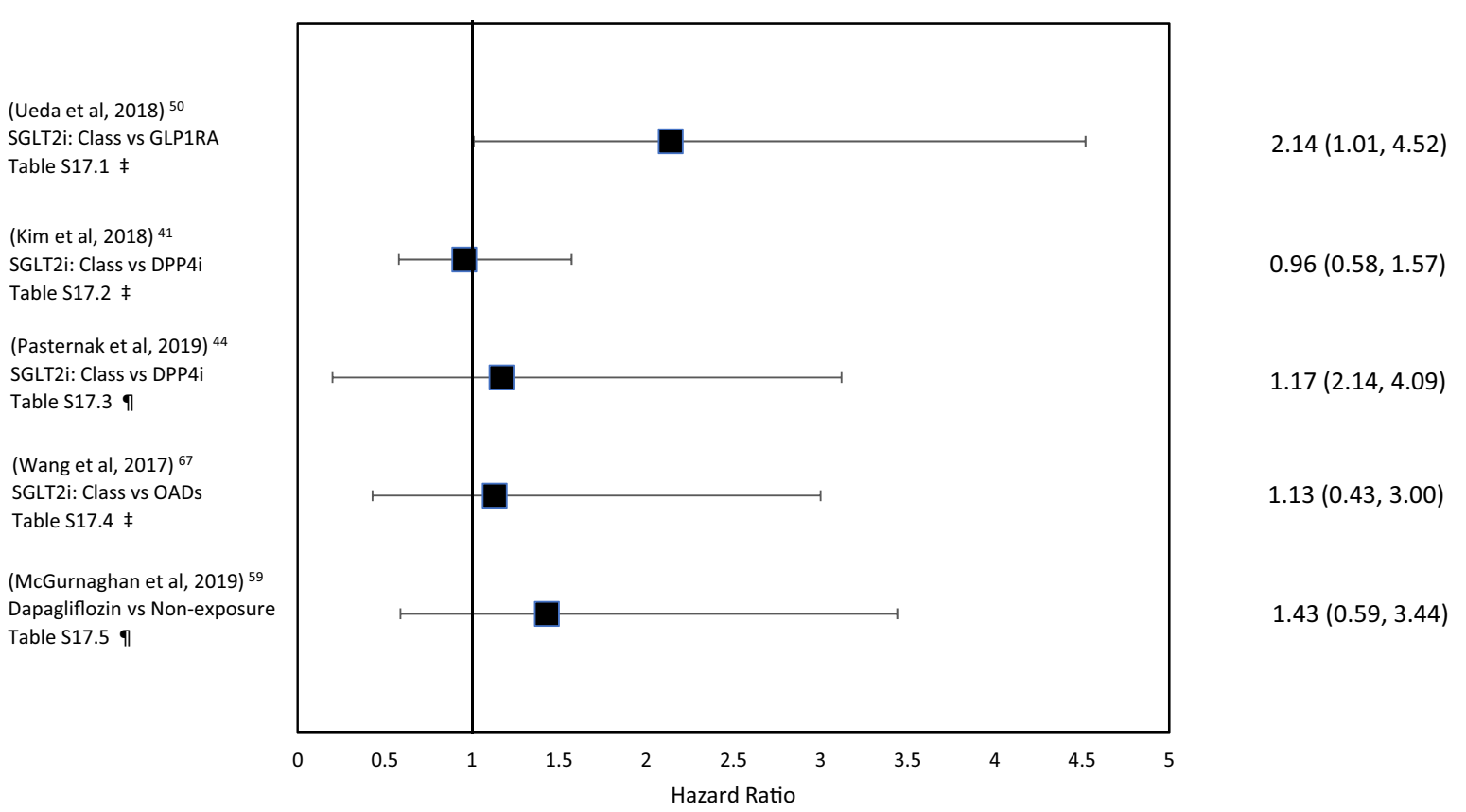

Fig. 4 DKA

might extend to primary as well as secondary CVD prevention (a key area of uncertainty in the CVOTs) $[54,57,58]$. The majority of studies we identified exploring HF/HHF suggest that SGLT2i exposure is associated with a reduction in this outcome. These findings confirm and extend HF reduction as a major benefit of SGLT2i exposure to populations with broader baseline HF and CVD risk profiles. It follows that the effect of SGLT2i on CVD is differential depending on the outcome being observed, where almost all studies exploring HF/HHF reported a negative association with this outcome whereas only half the studies exploring MI showed an associated reduction. However, this is not unexpected, where RCT data shows that all SGLT2is reduce HHF whereas none reduce $\mathrm{MI}$.

Whilst it is challenging to infer what proportion of the populations, in the studies we found, would have been eligible for inclusion in the CVOTs, examination of the baseline IHD suggests that the studies identified included a larger proportion of people at lower CVD risk.
The studies we identified suggest a neutral or reduced association with mortality (CV and ACM).

An area of controversy is whether or not the CVD benefits of SGLT2 inhibition extend to those with better glycaemic control than the inclusion criteria in the CVOTs. The DAPA-HF trial showed CV benefit in those with and without T2D [4]. However our included studies which adjusted for baseline HbA1c returned neutral associations for CVD outcomes $[45,49,62]$, except one [34].

We were unable to assess whether SGLT2i ought to be introduced earlier in the treatment of T2D as no studies explored this, likely because SGLT2is are still recommended as '> 1st-line' therapy.

\section{Safety Outcomes/Adverse Events}

The current regulatory position on SGLT2i safety is as follows. EMA has issued SGLT2i classwide warnings for DKA and amputation $[73,74]$. Medicines and Healthcare products 
Study details

(Ueda et al, 2018) ${ }^{50}$

SGLT2i: Class vs GLP1RA

Table S18.1 ‡

(Schmedt et al, 2018) ${ }^{46}$ Metformin plus SGLTi: Class vs AHA

Table S18.2 ¥

(Fralick et al, 2019) 38

Canagliflozin vs GLP1RA

Table S18.3 ¥

(Toulis et al, 2018) ${ }^{48}$

Dapagliflozin vs Unexposed

Table S18.4 ๆ

\section{Fracture}

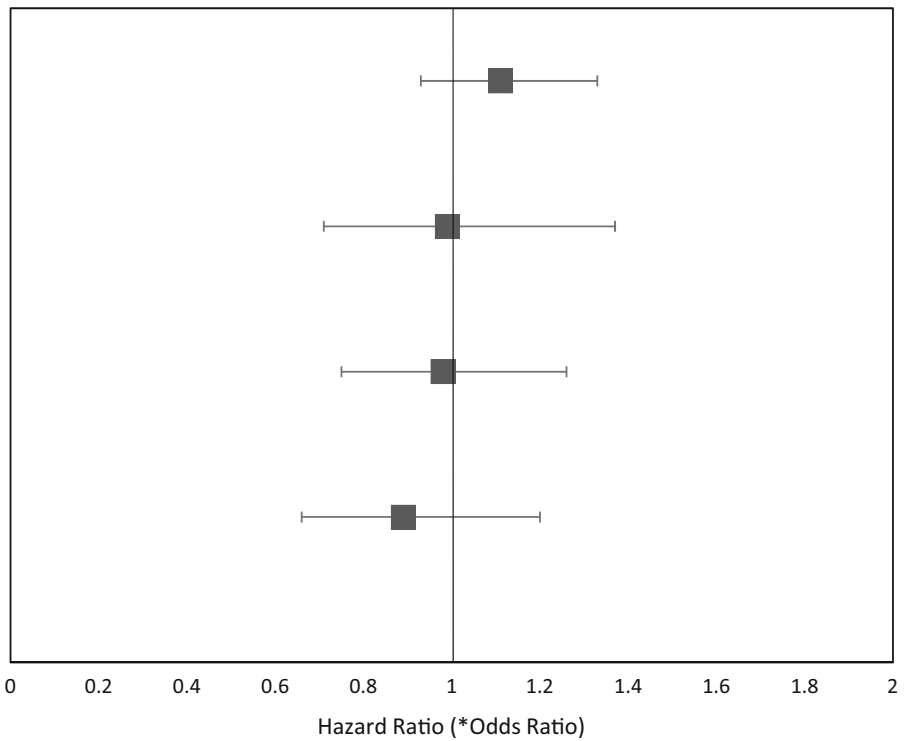

Point estimate $(95 \% \mathrm{Cl})$

$1.11(0.93,1.33)$

$0.99(0.71,1.37)^{*}$

$0.98(0.75,1.26)$

$0.89(0.66,1.2)$

Fig. 5 Bone fracture

Study details

(Ueda et al, 2018) 50

SGLT2i: Class vs GLP1RA

Table S19.1 ¥

(Dave et al, 2019) ${ }^{36}$

SGLT2i: Class vs GLP1RA

Table S19.2 ¥

(Dave et al, 2019) ${ }^{36}$

SGLT2i: Class vs DPP4

Table S19.2 ‡

(Gadzhanova et al, 2017) ${ }^{39}$

SGLT2i vs DPP4i

Table S19.3 ¥

(Lega et al, 2019) ${ }^{42}$

SGLT2i vs DPP4
Table S19.4 थ

(Amos et al, 2016) ${ }^{53}$

Canagliflozin vs Non-SGLT2i AHAs Table $\$ 19.6 \neq$
Urinary Tract Infection

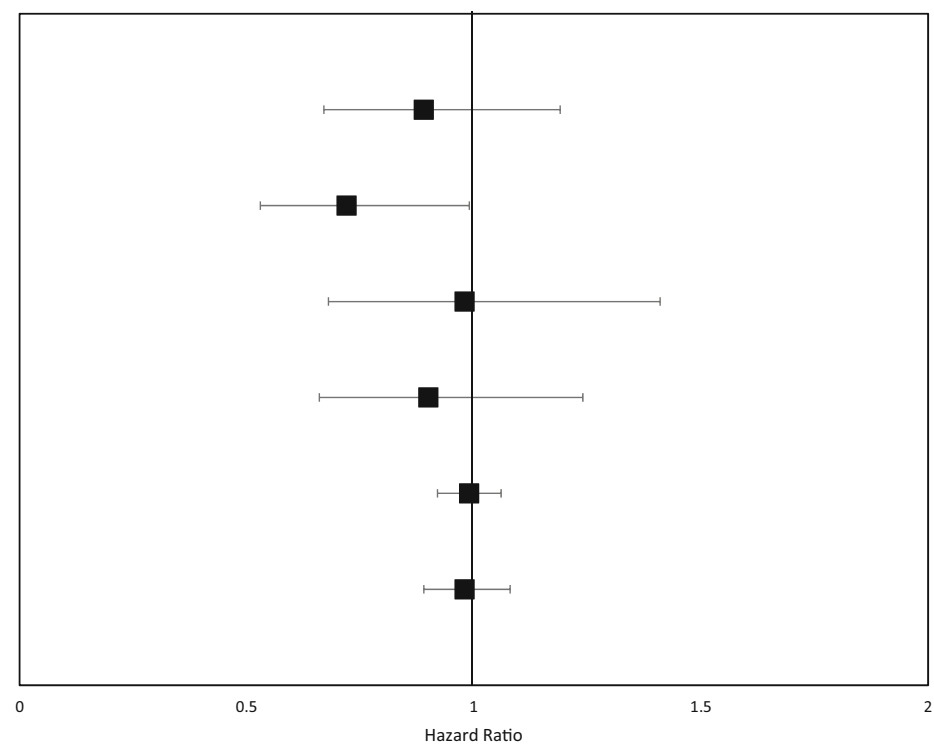

Point estimate $(95 \% \mathrm{Cl})$

\author{
$0.89(0.67,1.19)$ \\ $0.72(0.53,0.99)$ \\ $0.98(0.68,1.41)$ \\ $0.9(0.66,1.24)$ \\ $0.99(0.92,1.06)$ \\ $0.98(0.89,1.08)$
}

Fig. 6 Urinary tract infection

Regulatory Agency (MHRA) and FDA have issued class-wide warnings for Fournier's gangrene $[75,76]$. FDA has recently removed a warning for amputation from canagliflozin [77] (the only SGLT2i with this warning) but continues to warn about AKI for dapagliflozin and canagliflozin only [69] and for DKA and UTI class-wide [78]. The British National Formulary 


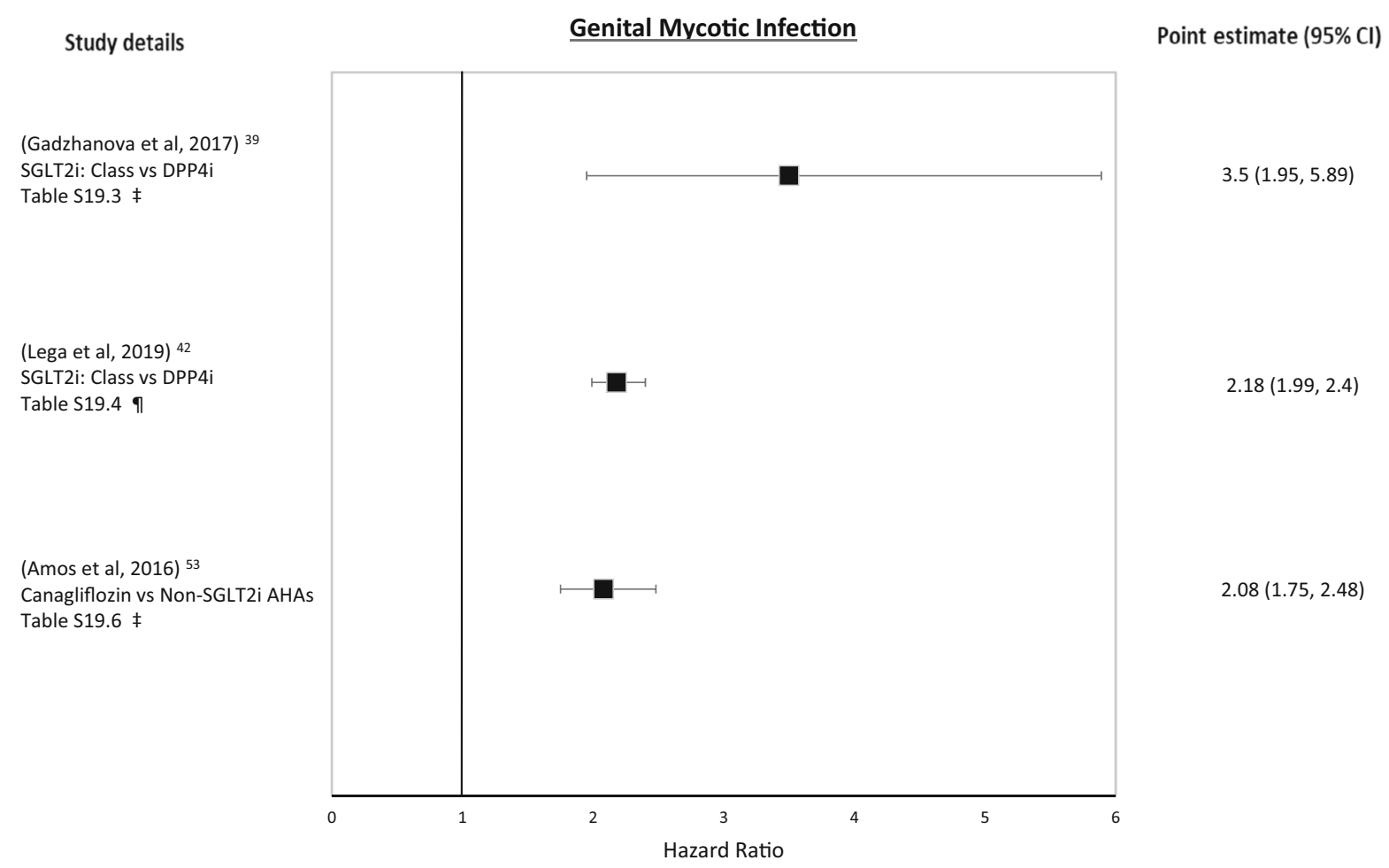

Fig. 7 Genital mycotic infection

(BNF) recommends monitoring renal function before and periodically after SGLT2i treatment initiation [79].

The studies we found did not suggest an increased risk of AKI (in any definition of AKI employed) [34, 43, 50].

On balance, there appears to be a signal for elevated LLA risk following SGLT2i exposure, but with considerable uncertainty $[32,35,37,44,50,52,59,68]$. Studies with longer follow-up are needed to address this uncertainty and to better identify subgroups at risk of amputation harm, if at all. Since LLA can occur at different levels and be a recurrent event, which in some cases might occur in quick succession in the same individual, amputation can be a challenging outcome to capture. However, most studies we identified either explored incident events $[35,37,59,66,68]$ (and censored on amputation occurrence) or conducted sensitivity analysis excluding prevalent amputation (which did not change the effect estimate) [33, 50, 52]. Only one study included prevalent amputation and did not adjust for this (but did include prior amputation in the propensity score) [44]. We believe that both users and clinicians should remain vigilant for, and regulators should continue to warn about, this adverse event.

From the studies identified $[41,44,50,59,67]$ there is considerable uncertainty as to the association of SGLT2is with DKA and clinicians and users ought to have a high index of suspicion for this outcome particularly in hospitalised patients (MHRA currently recommends 'interrupt treatment with SGLT2i in patients who are hospitalised for major surgery or acute serious illnesses' [80]). The gold standard for identification of DKA is through biochemical parameters, but the studies we identified used ICD or Systematized Nomenclature of Medicine Clinical Terms (SNOMED) codes giving rise to the possibility of misclassification. The role of incomplete DKA case ascertainment, intercurrent illness and insulin exposure (especially rapid dose reduction following post-SGLT2i exposure) ought to be explored further as must the possibility of cases 

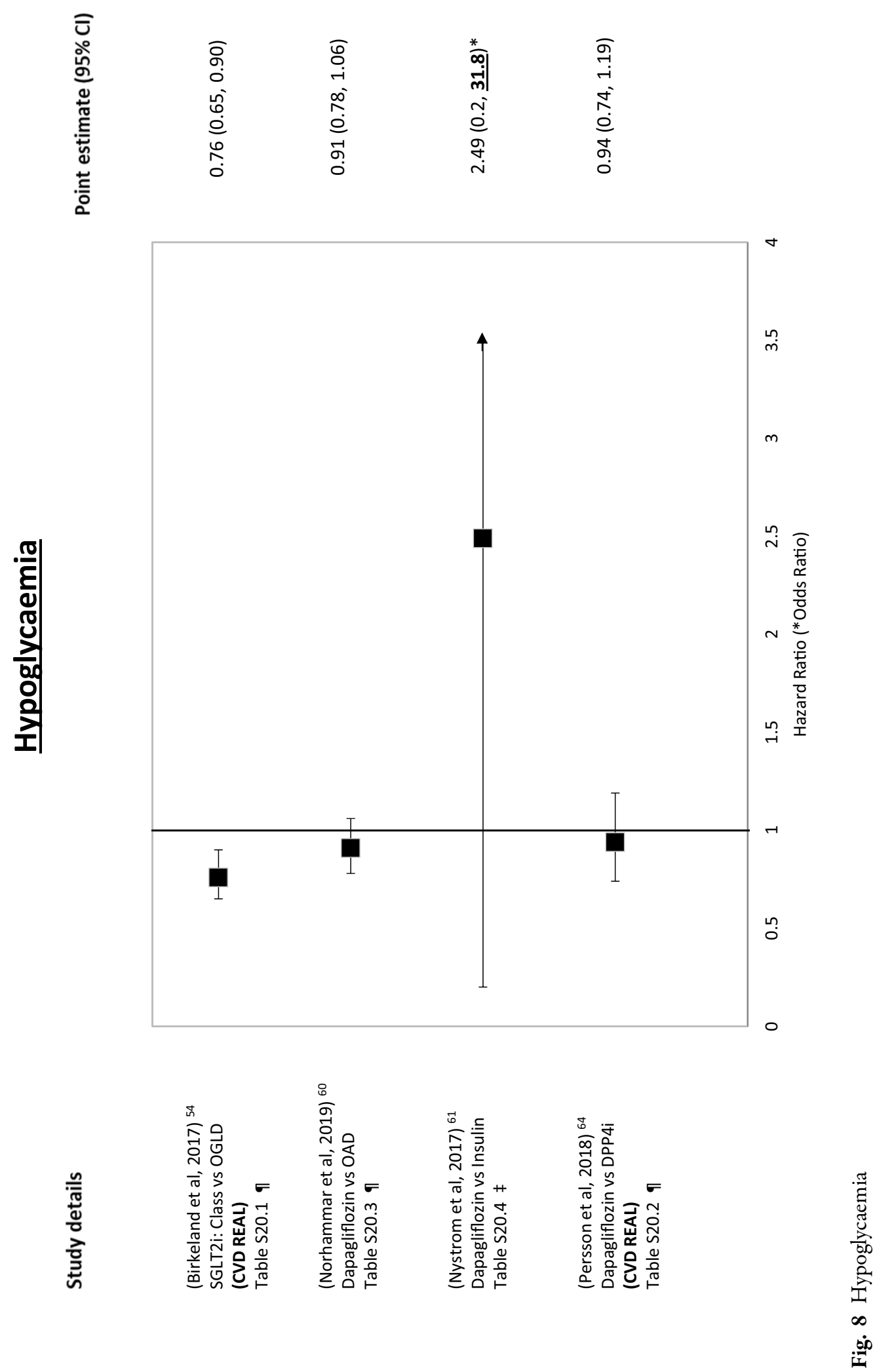
being misclassified as type 1 diabetes mellitus (T1D). We believe regulators should continue to warn about this adverse effect.

Although an association of SGLT2i with fractures cannot be ruled out, the evidence we found is reassuring $[38,46,48,50]$. However, if the mechanism of fracture is due to reduced bone mineral density [10] (rather than falls caused by hypovolaemia, which would occur rapidly after exposure) then it remains possible that insufficient person-years' exposure have accumulated to detect fragility fractures.

Although diabetes itself appears to elevate the risk of UTI [81], the associated risk following SGLT2i exposure appears minimal from the studies we found [36, 39, 42, 50, 53].

On the basis of these data, SGLT2i exposure does appear to be associated with increased risk of GMI in both men and women, likely due to the glycosuria caused by SGLT2is [39, 42, 53]. Users should be warned of the risk of this adverse effect.

Given that SGLT2i agents are insulin-independent, it is not surprising that they do not appear to be associated with hypoglycaemia $[54,60,61,64]$. However, it is important to understand, when SGLT2i agents are added to therapy that does cause hypoglycaemia (e.g. insulin and SUs), whether exposure increases or decreases the risk and this question remains unanswered by the data we found.

\section{Strengths and Limitations}

To our knowledge, this is the first systematic review of population-based observational studies looking at effectiveness in terms of $\mathrm{CV} /$ mortality outcomes and safety of SGLT2i agents in T2D. We identified studies from many countries and involved informationists to use appropriate search terms. The quality of the included studies is reported.

Given the heterogeneity of the studies both in terms of outcome definition, population, confounding control and analysis method, making generalised conclusions about these data is challenging.

As with all observational data exploring interventions, the studies included may be subject to unmeasured confounding and bias, particularly misclassification bias [27]. Observational pharmacoepidemiology is also subject to allocation bias/confounding-by-indication, although most of the included studies used modern methods to control for this (e.g. propensity score-matched, PSM). Nevertheless, the effectiveness estimates may be biased or confounded both in terms of direction and order of magnitude and in some cases may be entirely explained away by these; cautious interpretation is required.

For effectiveness studies, when observational pharmacoepidemiology studies conflict with RCT data, they should be viewed as hypothesisgenerating and the trial results preferred. RCTs often do not include a hypothesis for harm, nor are they powered to detect safety [27]. For safety, observational pharmacoepidemiology remains an important tool to enumerate the risks, especially when RCTs allude to an increased risk of harm due to numerical imbalances (which could be true or due to chance).

There was over-representation of certain data sources, e.g. Truven Health Analytics, which depending on the comparison and analysis methods chosen sometimes yielded different results for the same outcome, e.g. [33, 35].

A major challenge was the variety of composite endpoints in the studies identified, particularly for CVD, making comparisons challenging. While composite primary endpoints are useful for increasing the power of a study to detect change (by increasing the events counted towards the primary endpoint) it would be helpful if future studies emulated the composite outcomes reported in the CVOTs [82]. This would be useful for comparing identified studies between each other and also to RCT data.

We identified studies with potentially useful data in the form of letters to journals, which was one of our exclusion criteria (as it is unclear if these studies underwent peer review) [83-85]. In the case of Dave's letter, this identifies Fournier's gangrene as a potential complication of SGLT2i exposure [84]. This adverse event is warned about by both MHRA and FDA $[75,76]$. We deliberately excluded disproportionality reporting ratio studies based on interrogating 
adverse event reporting databases because of the high risk of bias, especially once an adverse event becomes known. However, skin photosensitivity [86] and Fournier's gangrene [87] both emerged as potential harms from disproportionality reporting ratio studies and should continue to be monitored for. There was a lack of studies identified exploring bladder and breast cancer, which are known concerns regarding SGLT2i exposure [88].

Many of the studies were commercially funded, which raises the possibility of commercial bias.

Despite considerable effort on our part to identify eligible studies systematically (including the manual searching of references), it remains possible that we overlooked potentially suitable studies and we omitted non-Englishlanguage publications because of resource limitation. Although the search terms we employed should detect observational studies conforming to the Strengthening the Reporting of OBservational studies in Epidemiology (STROBE) items on publication title and abstract, studies not conforming would be missed (although non-conforming could be considered a marker of poor quality).

Meta-analytic methods were not utilised in this study because of axiomatic breaches of the assumptions underpinning these, such as diversity of comparators, studies undertaken in the same data sources (leading to double counting of events) and a wide variety of methodologies rendering the associated treatment effect estimate uncombinable. We were keen not to give a false illusion of precision.

\section{CONCLUSION}

This systematic review provides a comprehensive view of the association of SGLT2is on a wide variety of $\mathrm{CV} /$ mortality effectiveness and safety outcomes in broad and unselected populations, generalisable to day-to-day practice. The 37 population-based studies included in the review confirm and contribute to the growing evidence that SGLT2is appear safe from the CVD perspective. They demonstrate that there may be an associated benefit in primary and secondary prevention of CVD events, particularly those related to HF. However, SGLT2i exposure may be associated with an increased risk of GMI, LLA and DKA, although longer follow-up studies are required to confirm this. Lastly, they showed no clear relationship between other safety events associated with their use (fractures, hypoglycaemia and UTI) but there may be evidence to support a reduced risk of AKI. These findings should be of interest to people living with $\mathrm{T} 2 \mathrm{D}$, their care providers and healthcare policymakers.

\section{ACKNOWLEDGEMENTS}

We would like to thank Ruth Jenkins at the University of Edinburgh Library and the Information and Resource Department of the Defence Medical Academy for their assistance with searching. Thomas M. Caparrotta acknowledges the support of Diabetes UK via his 'Sir George Alberti' fellowship: 18/005786. Rebecca M. Reynolds acknowledges the support of the British Heart Foundation (RE/18/5/ 34216).

Funding. No funding or sponsorship was received for this study or publication of this article. The Rapid Service Fee will be paid for out of funds from the University of Edinburgh set aside for this purpose

Authorship. All named authors meet the International Committee of Medical Journal Editors (ICMJE) criteria for authorship for this article, take responsibility for the integrity of the work as a whole, and have given their approval for this version to be published.

Authorship Contributions. Thomas M. Caparrotta and Andrew M. Greenhalgh conceived, designed and executed this study. Karen Osinski and Svenja Moser provided critical manuscript revision and restructuring, figure and table construction and approved the final version. Robert M. Gifford helped conceive the study question, contributed to the methods, provided critical input to the manuscript and 
approved the final version. Sarah $\mathrm{H}$. Wild and Rebecca M. Reynolds critically reviewed the draft manuscript and approved the final version. David J. Webb provided manuscript review/editing, expert clinical pharmacology opinion and research supervision. Helen M. Colhoun provided expert pharmacoepidemiology opinion and research supervision.

Disclosures. Thomas M. Caparrotta, Andrew M. Greenhalgh, Karen Osinski, Robert M. Gifford, Svenja Moser, Sarah H. Wild, Rebecca M. Reynolds, David J. Webb, Helen M. Colhoun report no disclosures and no conflicts of interest.

Compliance with Ethics Guidelines. This article is based on previously conducted studies and does not contain any new studies with human participants or animals performed by any of the authors.

Data Availability. All data generated or analysed during this study are included in this published article/as supplementary information files.

Open Access. This article is licensed under a Creative Commons Attribution-NonCommercial 4.0 International License, which permits any non-commercial use, sharing, adaptation, distribution and reproduction in any medium or format, as long as you give appropriate credit to the original author(s) and the source, provide a link to the Creative Commons licence, and indicate if changes were made. The images or other third party material in this article are included in the article's Creative Commons licence, unless indicated otherwise in a credit line to the material. If material is not included in the article's Creative Commons licence and your intended use is not permitted by statutory regulation or exceeds the permitted use, you will need to obtain permission directly from the copyright holder. To view a copy of this licence, visit http:// creativecommons.org/licenses/by-nc/4.0/.

\section{REFERENCES}

1. Verma S, McMurray JJV. SGLT2 inhibitors and mechanisms of cardiovascular benefit: a state-ofthe-art review. Diabetologia. 2018;61:2108-17.

2. Zinman B, Wanner C, Lachin JM, et al. Empagliflozin, cardiovascular outcomes, and mortality in type 2 diabetes. NEJM. 2015;373:2117-28.

3. Wiviott SD, Raz I, Bonaca MP, et al. Dapagliflozin and cardiovascular outcomes in type 2 diabetes. NEJM. 2019;380:347-57.

4. McMurray JJV, Solomon SD, Inzucchi SE, et al. Dapagliflozin in patients with heart failure and reduced ejection fraction. NEJM. 2019;381: 1995-2008.

5. Neal B, Perkovic V, Mahaffey KW, et al. Canagliflozin and cardiovascular and renal events in type 2 diabetes. NEJM. 2017;377:644-57.

6. Perkovic V, Jardine MJ, Neal B, et al. Canagliflozin and renal outcomes in type 2 diabetes and nephropathy. NEJM. 2019;380:2295-306.

7. Alba M, Xie J, Fung A, Desai M. The effects of canagliflozin, a sodium glucose co-transporter 2 inhibitor, on mineral metabolism and bone in patients with type 2 diabetes mellitus. Curr Med Res Opin. 2016;32:1375-85.

8. Bailey CJ, Gross JL, Hennicken D, Iqbal N, Mansfield TA, List JF. Dapagliflozin add-on to metformin in type 2 diabetes inadequately controlled with metformin: a randomized, double-blind, placebocontrolled 102-week trial. BMC Med. 2013;11:43.

9. Bersoff-Matcha SJ, Chamberlain C, Cao C, Kortepeter $\mathrm{C}$, Chong WH. Fournier gangrene associated with sodium-glucose cotransporter-2 inhibitors. Ann Intern Med. 2019;170:764-9.

10. Bilezikian JP, Watts NB, Usiskin K, et al. Evaluation of bone mineral density and bone biomarkers in patients with type 2 diabetes treated with canagliflozin. J Clin Endocrinol Metab. 2016;101:44-51.

11. Cefalu WT, Leiter LA, Yoon K-H, et al. Efficacy and safety of canagliflozin versus glimepiride in patients with type 2 diabetes inadequately controlled with metformin (CANTATA-SU): 52 week results from a randomised, double-blind, phase 3 non-inferiority trial. Lancet. 2013;382:941-50.

12. Clar C, Gill JA, Court R, Waugh N. Systematic review of SGLT2 receptor inhibitors in dual or triple therapy in type 2 diabetes. BMJ Open. 2012;2: e001007. 
13. Girman CJ, Kou TD, Cai B, et al. Patients with type 2 diabetes mellitus have higher risk for acute pancreatitis compared with those without diabetes. Diabetes Obes Metab. 2010;12:766-71.

14. Johnsson KM, Ptaszynska A, Schmitz B, Sugg J, Parikh SJ, List JF. Urinary tract infections in patients with diabetes treated with dapagliflozin. J Diabetes Complicat. 2013;27:473-8.

15. Khouri C, Cracowski J-L, Roustit M. SGLT-2 inhibitors and the risk of lower-limb amputation: is this a class effect? Diabetes Obes Metab. 2018;20: 1531-4.

16. Kohler S, Kaspers S, Salsali A, Zeller C, Woerle HJ. Analysis of fractures in patients with type 2 diabetes treated with empagliflozin in pooled data from placebo-controlled trials and a head-to-head study versus glimepiride. Diabetes Care. 2018;41: 1809-16.

17. Kumar S, Costello AJ, Colman PG. Fournier's gangrene in a man on empagliflozin for treatment of type 2 diabetes. Diabet Med. 2017;34:1646-8.

18. Ljunggren Ö, Bolinder J, Johansson L, et al. Dapagliflozin has no effect on markers of bone formation and resorption or bone mineral density in patients with inadequately controlled type 2 diabetes mellitus on metformin. Diabetes Obes Metab. 2012;14: 990-9.

19. Musso G, Gambino R, Cassader M, Pagano G. A novel approach to control hyperglycemia in type 2 diabetes: sodium glucose co-transport (SGLT) inhibitors: systematic review and meta-analysis of randomized trials. Ann Med. 2012;44:375-93.

20. Nauck MA, Prato SD, Meier JJ, et al. Dapagliflozin versus glipizide as add-on therapy in patients with type 2 diabetes who have inadequate glycemic control with metformin: a randomized, 52-week, double-blind, active-controlled noninferiority trial. Diabetes Care. 2011;34:2015-22.

21. Noel RA, Braun DK, Patterson RE, Bloomgren GL. Increased risk of acute pancreatitis and biliary disease observed in patients with type 2 diabetes: a retrospective cohort study. Diabetes Care. 2009;32: 834-8.

22. Nyirjesy P, Zhao Y, Ways K, Usiskin K. Evaluation of vulvovaginal symptoms and Candida colonization in women with type 2 diabetes mellitus treated with canagliflozin, a sodium glucose co-transporter 2 inhibitor. Curr Med Res Opin. 2012;28: 1173-8.

23. Peters AL, Buschur EO, Buse JB, Cohan P, Diner JC, Hirsch IB. Euglycemic diabetic ketoacidosis: a potential complication of treatment with sodium-glucose cotransporter 2 inhibition. Diabetes Care. 2015;38:1687-93.

24. Tang HL, Li DD, Zhang JJ, et al. Lack of evidence for a harmful effect of sodium-glucose co-transporter 2 (SGLT2) inhibitors on fracture risk among type 2 diabetes patients: a network and cumulative metaanalysis of randomized controlled trials. Diabetes Obes Metab. 2016;18:1199-206.

25. Watts NB, Bilezikian JP, Usiskin K, et al. Effects of canagliflozin on fracture risk in patients with type 2 diabetes mellitus. J Clin Endocrinol Metab. 2016;101:157-66.

26. Wilding JPH, Woo V, Soler NG, et al. Long-term efficacy of dapagliflozin in patients with type 2 diabetes mellitus receiving high doses of insulin. Ann Intern Med. 2012;156:405-15.

27. Caparrotta TM, Dear JW, Colhoun HM, Webb DJ. Pharmacoepidemiology: using randomised control trials and observational studies in clinical decisionmaking. Br J Clin Pharmacol. 2019;85:1907-24.

28. Downs SH, Black N. The feasibility of creating a checklist for the assessment of the methodological quality both of randomised and non-randomised studies of health care interventions. J Epidemiol Community Health. 1998;52:377-84.

29. Hootman JM, Driban JB, Sitler MR, Harris KP, Cattano NM. Reliability and validity of three quality rating instruments for systematic reviews of observational studies. Res Synth Methods. 2011;2:110-8.

30. Berlin JA, Crowe BJ, Xia HA, Evans SJW. The use of metaanalysis in pharmacoepidemiology. In: Pharmacoepidemiology. New Jersey: Wiley; 2019. p. 897-947.

31. Page MJ, Higgins JPT, Sterne JAC. Chapter 13: assessing risk of bias due to missing results in a synthesis. In: Higgins JPT, Thomas J, Chandler J, Cumpston M, Li T, Page MJ, Welch VA, editors. Cochrane Handbook for Systematic Reviews of Interventions. 2020. https://training.cochrane.org/ handbook/current/chapter-13. Accessed 29 Jul 2020.

32. Adimadhyam S, Schumock GT, Calip GS, Marsh DES, Layden BT, Lee TA. Increased risk of mycotic infections associated with sodium-glucose cotransporter 2 inhibitors: a prescription sequence symmetry analysis. Br J Clin Pharmacol. 2019;85: 160-8.

33. Adimadhyam S, Lee TA, Calip GS, Marsh DES, Layden BT, Schumock GT. Risk of amputations associated with SGLT2 inhibitors compared to DPP4 inhibitors: a propensity-matched cohort study. Diabetes Obes Metab. 2018;20:2792-9. 
34. Cahn A, Melzer-Cohen C, Pollack R, Chodick G, Shalev V. Acute renal outcomes with sodium-glucose co-transporter-2 inhibitors: real-world data analysis. Diabetes Obes Metab. 2019;21:340-8.

35. Chang H-Y, Singh S, Mansour O, Baksh S, Alexander GC. Association between sodium-glucose cotransporter 2 inhibitors and lower extremity amputation among patients with type 2 diabetes. JAMA Intern Med. 2018;178:1190-8.

36. Dave CV, Schneeweiss S, Kim D, Fralick M, Tong A, Patorno E. Sodium-glucose cotransporter-2 inhibitors and the risk for severe urinary tract infections. Ann Intern Med. 2019;171:248-56.

37. Dawwas GK, Smith SM, Park H. Cardiovascular outcomes of sodium glucose cotransporter-2 inhibitors in patients with type 2 diabetes. Diabetes Obes Metab. 2019;21:28-36.

38. Fralick M, Kim SC, Schneeweiss S, Kim D, Redelmeier DA, Patorno E. Fracture risk after initiation of use of canagliflozin: a cohort study. Ann Intern Med. 2019;170:155.

39. Gadzhanova S, Pratt N, Roughead E. Use of SGLT2 inhibitors for diabetes and risk of infection: analysis using general practice records from the NPS MedicineWise MedicineInsight program. Diabetes Res Clin Pract. 2017;130:180-5.

40. Kim Y-G, Han SJ, Kim DJ, Lee K-W, Kim HJ. Association between sodium glucose co-transporter 2 inhibitors and a reduced risk of heart failure in patients with type 2 diabetes mellitus: a real-world nationwide population-based cohort study. Cardiovasc Diabetol. 2018;17:91.

41. Kim Y-G, Jeon JY, Han SJ, Kim DJ, Lee K-W, Kim HJ. Sodium-glucose co-transporter-2 inhibitors and the risk of ketoacidosis in patients with type 2 diabetes mellitus: a nationwide population-based cohort study. Diabetes Obes Metab. 2018;20:1852-8.

42. Lega IC, Bronskill SE, Campitelli MA, et al. Sodium glucose cotransporter 2 inhibitors and risk of genital mycotic and urinary tract infection: a population-based study of older women and men with diabetes. Diabetes Obes Metab. 2019;21:2394-404.

43. Nadkarni GN, Ferrandino R, Chang A, et al. Acute kidney injury in patients on SGLT2 inhibitors: a propensity-matched analysis. Diabetes Care. 2017;40:1479-85.

44. Pasternak B, Ueda P, Eliasson B, et al. Use of sodium glucose cotransporter 2 inhibitors and risk of major cardiovascular events and heart failure: Scandinavian register based cohort study. BMJ. 2019;366: 14772.
45. Patorno E, Goldfine AB, Schneeweiss S, et al. Cardiovascular outcomes associated with canagliflozin versus other non-gliflozin antidiabetic drugs: population based cohort study. BMJ. 2018;360:k119.

46. Schmedt N, Andersohn F, Walker J, Garbe E. Sodium-glucose co-transporter-2 inhibitors and the risk of fractures of the upper or lower limbs in patients with type 2 diabetes: a nested case-control study. Diabetes Obes Metab. 2019;21:52-60.

47. Shao S-C, Chang K-C, Hung M-J, et al. Comparative risk evaluation for cardiovascular events associated with dapagliflozin vs. empagliflozin in real-world type 2 diabetes patients: a multi-institutional cohort study. Cardiovasc Diabetol. 2019;18:120.

48. Toulis KA, Bilezikian JP, Thomas GN, et al. Initiation of dapagliflozin and treatment-emergent fractures. Diabetes Obes Metab. 2018;20:1070-4.

49. Toulis KA, Willis BH, Marshall T, et al. All-cause mortality in patients with diabetes under treatment with dapagliflozin: a population-based, open-cohort study in The Health Improvement Network database. J Clin Endocrinol Metab. 2017;102: 1719-25.

50. Ueda P, Svanström H, Melbye $M$, et al. Sodium glucose cotransporter 2 inhibitors and risk of serious adverse events: nationwide register based cohort study. BMJ. 2018;363:k4365.

51. Weeda ER, Cassarly C, Brinton DL, Shirley DW, Simpson KN. Loop diuretic use among patients with heart failure and type 2 diabetes treated with sodium glucose cotransporter-2 inhibitors. J Diabetes Complicat. 2019;33:567-71.

52. Yang JY, Wang T, Pate V, et al. Sodium-glucose cotransporter-2 inhibitor use and risk of lower-extremity amputation: evolving questions, evolving answers. Diabetes Obes Metab. 2019;21:1223-36.

53. Amos TB, Montejano L, Juneau P, Bolge SC. Healthcare costs of urinary tract infections and genital mycotic infections among patients with type 2 diabetes mellitus initiated on canagliflozin: a retrospective cohort study. J Med Econ. 2017;20: 303-13.

54. Birkeland KI, Jørgensen ME, Carstensen B, et al. Cardiovascular mortality and morbidity in patients with type 2 diabetes following initiation of sodium-glucose co-transporter-2 inhibitors versus other glucose-lowering drugs (CVD-REAL Nordic): a multinational observational analysis. Lancet Diabetes Endocrinol. 2017;5:709-17.

55. Gautam S, Agiro A, Barron J, Power T, Weisman H, White J. Heart failure hospitalization risk associated with use of two classes of oral antidiabetic 
medications: an observational, real-world analysis. Cardiovasc Diabetol. 2017;16:93.

56. Kosiborod M, Lam CSP, Kohsaka S, et al. Cardiovascular events associated with SGLT-2 inhibitors versus other glucose-lowering drugs: the CVDREAL 2 study. J Am Coll Cardiol. 2018;71:2628-39.

57. Kosiborod M, Birkeland KI, Cavender MA, et al. Rates of myocardial infarction and stroke in patients initiating treatment with SGLT2-inhibitors versus other glucose-lowering agents in real-world clinical practice: results from the CVD-REAL study. Diabetes Obes Metab. 2018;20:1983-7.

58. Kosiborod M, Cavender MA, Fu AZ, et al. Lower risk of heart failure and death in patients initiated on sodium-glucose cotransporter-2 inhibitors versus other glucose-lowering drugs. Circulation. 2017;136:249-59.

59. McGurnaghan SJ, Brierley L, Caparrotta TM, et al. The effect of dapagliflozin on glycaemic control and other cardiovascular disease risk factors in type 2 diabetes mellitus: a real-world observational study. Diabetologia. 2019;62:621-32.

60. Norhammar A, Bodegård J, Nyström T, Thuresson M, Nathanson D, Eriksson JW. Dapagliflozin and cardiovascular mortality and disease outcomes in a population with type 2 diabetes similar to that of the DECLARE-TIMI 58 trial: a nationwide observational study. Diabetes Obes Metab. 2019;21: 1136-45.

61. Nyström T, Bodegard J, Nathanson D, Thuresson M, Norhammar A, Eriksson JW. Novel oral glucoselowering drugs are associated with lower risk of allcause mortality, cardiovascular events and severe hypoglycaemia compared with insulin in patients with type 2 diabetes. Diabetes Obes Metab. 2017;19:831-41.

62. O'Brien MJ, Karam SL, Wallia A, et al. Association of second-line antidiabetic medications with cardiovascular events among insured adults with type 2 diabetes. JAMA Netw Open. 2018;1: e186125-e186125.

63. Patorno E, Pawar A, Franklin JM, et al. Empagliflozin and the risk of heart failure hospitalization in routine clinical care. Circulation. 2019;139: 2822-30.

64. Persson F, Nyström T, Jørgensen ME, et al. Dapagliflozin is associated with lower risk of cardiovascular events and all-cause mortality in people with type 2 diabetes (CVD-REAL Nordic) when compared with dipeptidyl peptidase- 4 inhibitor therapy: a multinational observational study. Diabetes Obes Metab. 2018;20:344-51.
65. Ryan PB, Buse JB, Schuemie MJ, et al. Comparative effectiveness of canagliflozin, SGLT2 inhibitors and non-SGLT2 inhibitors on the risk of hospitalization for heart failure and amputation in patients with type 2 diabetes mellitus: a real-world meta-analysis of 4 observational databases (OBSERVE-4D). Diabetes Obes Metab. 2018;20:2585-97.

66. Udell JA, Yuan Z, Rush T, Sicignano NM, Galitz M, Rosenthal N. Cardiovascular outcomes and risks after initiation of a sodium glucose cotransporter 2 inhibitor. Circulation. 2018;137:1450-9.

67. Wang Y, Desai M, Ryan PB, et al. Incidence of diabetic ketoacidosis among patients with type 2 diabetes mellitus treated with SGLT2 inhibitors and other antihyperglycemic agents. Diabetes Res Clin Pract. 2017;128:83-90.

68. Yuan Z, DeFalco FJ, Ryan PB, et al. Risk of lower extremity amputations in people with type 2 diabetes mellitus treated with sodium-glucose cotransporter-2 inhibitors in the USA: a retrospective cohort study. Diabetes Obes Metab. 2018;20:582-9.

69. Food and Drug Administration. FDA Drug Safety Communication: FDA strengthens kidney warnings for diabetes medicines canagliflozin (Invokana, Invokamet) and dapagliflozin (Farxiga, Xigduo XR). FDA. 2019. https://www.fda.gov/drugs/drug-safetyand-availability/fda-drug-safety-communicationfda-strengthens-kidney-warnings-diabetesmedicines-canagliflozin. Accessed 3 Jul 2020

70. Zelniker TA, Wiviott SD, Raz I, et al. SGLT2 inhibitors for primary and secondary prevention of cardiovascular and renal outcomes in type 2 diabetes: a systematic review and meta-analysis of cardiovascular outcome trials. Lancet. 2019;393: $31-9$.

71. Wanner C, Inzucchi SE, Lachin JM, et al. Empagliflozin and progression of kidney disease in type 2 diabetes. NEJM. 2016;375:323-34.

72. Li D, Wang T, Shen S, Fang Z, Dong Y, Tang H. Urinary tract and genital infections in patients with type 2 diabetes treated with sodium-glucose cotransporter 2 inhibitors: a meta-analysis of randomized controlled trials. Diabetes Obes Metab. 2017;19:348-55.

73. European Medicines Agency. SGLT2 inhibitorsDKA. EMA. 2018. https://www.ema.europa.eu/en/ medicines/human/referrals/sglt2-inhibitors. Accessed 14 Oct 2020.

74. European Medicines Agency. SGLT2 inhibitors (previously canagliflozin)-amputation. EMA. 2018. https://www.ema.europa.eu/en/medicines/human/ referrals/sglt2-inhibitors-previously-canagliflozin. Accessed 14 Oct 2020. 
75. Food and Drug Administration. FDA warns about rare occurrences of a serious infection of the genital area with SGLT2 inhibitors for diabetes. FDA. 2019. https://www.fda.gov/drugs/drug-safety-andavailability/fda-warns-about-rare-occurrencesserious-infection-genital-area-sglt2-inhibitorsdiabetes. Accessed 14 Oct 2020.

76. SGLT2 inhibitors: reports of Fournier's gangrene (necrotising fasciitis of the genitalia or perineum). GOV.UK. 2020. https://www.gov.uk/drug-safetyupdate/sglt2-inhibitors-reports-of-fournier-sgangrene-necrotising-fasciitis-of-the-genitalia-orperineum. Accessed 14 Oct 2020.

77. Food and Drug Administration. FDA removes Boxed Warning about risk of leg and foot amputations for the diabetes medicine canagliflozin (Invokana, Invokamet, Invokamet XR). FDA. 2020. https:// www.fda.gov/drugs/drug-safety-and-availability/ fda-removes-boxed-warning-about-risk-leg-andfoot-amputations-diabetes-medicine-canagliflozin. Accessed 14 Oct 2020.

78. Food and Drug Administration. FDA revises labels of SGLT2 inhibitors for diabetes to include warnings about too much acid in the blood and serious urinary tract infections. FDA. 2020. https://www. fda.gov/drugs/drug-safety-and-availability/fdarevises-labels-sglt2-inhibitors-diabetes-includewarnings-about-too-much-acid-blood-and-serious. Accessed 14 Oct 2020.

79. BNF: British National Formulary-NICE. 2020. https://bnf.nice.org.uk/. Accessed 14 Oct 2020.

80. SGLT2 inhibitors: updated advice on the risk of diabetic ketoacidosis. GOV.UK. 2020. https://www. gov.uk/drug-safety-update/sglt2-inhibitorsupdated-advice-on-the-risk-of-diabeticketoacidosis. Accessed 19 Oct 2020.
81. Ribera MC, Pascual R, Orozco D, Pérez Barba C, Pedrera V, Gil V. Incidence and risk factors associated with urinary tract infection in diabetic patients with and without asymptomatic bacteriuria. Eur J Clin Microbiol Infect Dis. 2006;25:389-93.

82. Hernán MA, Robins JM. Using big data to emulate a target trial when a randomized trial is not available. Am J Epidemiol. 2016;183:758-64.

83. Abrahami D, Douros A, Yin H, Yu OHY, Azoulay L. Sodium-glucose cotransporter 2 inhibitors and the risk of fractures among patients with type 2 diabetes. Diabetes Care. 2019;42:e150-2.

84. Dave CV, Schneeweiss S, Patorno E. Association of sodium-glucose cotransporter 2 inhibitor treatment with risk of hospitalization for fournier gangrene among men. JAMA Intern Med. 2019;179: 1587-90.

85. Fralick M, Schneeweiss S, Patorno E. Risk of diabetic ketoacidosis after initiation of an SGLT2 inhibitor. N Engl J Med. 2017. https://doi.org/10.1056/ NEJMc1701990.

86. Raschi E, Parisotto M, Forcesi E, et al. Adverse events with sodium-glucose co-transporter-2 inhibitors: a global analysis of international spontaneous reporting systems. Nutr Metab Cardiovasc Dis. 2017;27:1098-107.

87. Fadini GP, Sarangdhar M, Ponti FD, Avogaro A, Raschi E. Pharmacovigilance assessment of the association between Fournier's gangrene and other severe genital adverse events with SGLT-2 inhibitors. BMJ Open Diabetes Res Care. 2019;7:e000725.

88. Scheen AJ. An update on the safety of SGLT2 inhibitors. Expert Opin Drug Saf. 2019;18:295-311. 